\title{
THE GROUPOIDS OF ADAPTABLE SEPARATED GRAPHS AND THEIR TYPE SEMIGROUPS
}

\author{
PERE ARA, JOAN BOSA, ENRIQUE PARDO, AND AIDAN SIMS \\ Dedicated to the memory of Emili Ara Aymami
}

\begin{abstract}
Given an adaptable separated graph, we construct an associated groupoid and explore its type semigroup. Specifically, we first attach to each adaptable separated graph a corresponding semigroup, which we prove is an $E^{*}$-unitary inverse semigroup. As a consequence, the tight groupoid of this semigroup is a Hausdorff étale groupoid. We show that this groupoid is always amenable, and that the type semigroups of groupoids obtained from adaptable separated graphs in this way include all finitely generated conical refinement monoids. The first three named authors will utilize this construction in forthcoming work to solve the Realization Problem for von Neumann regular rings, in the finitely generated case.
\end{abstract}

\section{INTRODUCTION.}

There has been significant recent interest in the structure of the type semigroup of an ample groupoid [12, 28, 29] and its influence in determining properties of the associated (reduced) groupoid $C^{*}$-algebra. In particular the stably finite versus purely infinite dichotomy has been established, under mild hypotheses in the above-mentioned papers, using the type semigroup. Additionally, the enveloping group of the type semigroup of an ample groupoid $\mathcal{G}$ is precisely the homology group $H_{0}(\mathcal{G})$ of the groupoid, and so the induced map $\operatorname{Typ}(\mathcal{G}) \rightarrow \mathcal{V}\left(C_{r}^{*}(\mathcal{G})\right)$ from $\operatorname{Typ}(\mathcal{G})$ to the Murray-von Neumann semigroup $\mathcal{V}\left(C_{r}^{*}(\mathcal{G})\right)$ can be viewed as a non-stable precursor of the natural map $H_{0}(\mathcal{G}) \rightarrow K_{0}\left(C_{r}^{*}(\mathcal{G})\right)$ appearing in the statement of Matui's Conjecture. In particular it is of great interest to determine the family of ample groupoids such that the map $\operatorname{Typ}(\mathcal{G}) \rightarrow \mathcal{V}\left(C_{r}^{*}(\mathcal{G})\right)$ and its stabilized sibling $H_{0}(\mathcal{G}) \rightarrow K_{0}\left(C_{r}^{*}(\mathcal{G})\right)$ are injective (see [24, 26, 27, 19]).

In this paper, we introduce a new class of ample Hausdorff groupoids associated to separated graphs, and describe concrete representations of their Steinberg algebras and $C^{*}$-algebras. This new class of groupoids generalizes the well-known graph groupoids of row-finite graphs [22], and are designed to have a more general type semigroup than those. Specifically, for a row-finite graph $E$, our methods demonstrate that the type semigroup of the associated graph

Date: April 11, 2019.

2010 Mathematics Subject Classification. Primary 16D70, Secondary 06F20, 19K14, 20K20, 46L05, 46L55.

Key words and phrases. Steinberg algebra, Refinement monoid, Type semigroup.

The first, second and third authors were partially supported by the DGI-MINECO and European Regional Development Fund, jointly, through the grant MTM2017-83487-P. The first and second authors acknowledge support from the Spanish Ministry of Economy and Competitiveness, through the María de Maeztu Programme for Units of Excellence in R\&D (MDM-2014-0445) and from the Generalitat de Catalunya through the grant 2017-SGR-1725. The third author was partially supported by PAI III grant FQM-298 of the Junta de Andalucía. The fourth author was partially supported by the Australian Research Council grant DP150101595. 
groupoid is canonically isomorphic to the graph monoid $M(E)$ introduced in [8]; that is, the commutative monoid with generators $\left\{a_{v}: v \in E^{0}\right\}$, and defining relations $a_{v}=\sum_{e \in s^{-1}(v)} a_{r(e)}$ whenever $v$ is not a sink in $E$. The graph monoids $M(E)$, as well as all the monoids $\operatorname{Typ}(\mathcal{G})$ of an ample groupoid $\mathcal{G}$, are refinement monoids, in the sense that they satisfy the Riesz refinement property. However, the class of graph monoids does not even cover the very natural class of all finitely generated conical refinement monoids. Indeed it was already shown in [11] that the finitely generated refinement monoid $\left\langle p, q_{1}, q_{2}: p+q_{1}=p=p+q_{2}\right\rangle$ is not a graph monoid; that is, it is not isomorphic to $M(E)$ for any row-finite graph $E$. In this paper we show that this problem can be solved by considering a special class of separated graphs, which we call adaptable separated graphs.

Our work is also motivated by the realization problem for von Neumann regular rings (see for instance $[3,20])$. Using the construction in this paper, the three first-named authors will show in [5] that every finitely generated conical refinement monoid arises as the monoid $\mathcal{V}\left(A_{K}(\mathcal{G}) \Sigma^{-1}\right)$ associated to a von Neumann regular ring of the form $A_{K}(\mathcal{G}) \Sigma^{-1}$ for a suitable universal localization of the Steinberg algebra $A_{K}(\mathcal{G})$, where $\mathcal{G}$ belongs to the class of groupoids constructed here and $K$ is an arbitrary field. In this respect, it is relevant the structure of a Steinberg algebra $A_{K}(\mathcal{G})$ of an ample groupoid $\mathcal{G}$ over a field $K$ [15, 34], which has received quite a bit of attention in the last few years, see for instance the survey papers $[16,31]$.

Recall from [7] that a separated graph is a pair $(E, C)$ where $E$ is a directed graph and $C=$ $\bigsqcup_{v \in E^{0}} C_{v}$ is a partition of $E^{1}$ which is finer than the partition induced by the source function $s: E^{1} \rightarrow E^{0}$. Given a finitely separated graph, that is, a separated graph such that all the sets in the partition $C$ are finite, the monoid $M(E, C)$ is defined in [7] as the commutative monoid with generators $\left\{a_{v}: v \in E^{0}\right\}$ and defining relations given by the equations $a_{v}=\sum_{x \in X} a_{r(x)}$ for $v \in E^{0}$ and $X \in C_{v}$. Unlike graph monoids, $M(E, C)$ is not always a refinement monoid, but sufficient conditions under which $M(E, C)$ is a refinement monoid were identified in [7, Section 5]. The key observation for the present paper is that a restricted class of separated graphs, which we have termed adaptable separated graphs, is enough for our purposes. In fact, for this family of separated graphs $(E, C)$, the refinement property for $M(E, C)$ does hold, and the monoids $M(E, C)$ belong to a especially well-behaved class of refinement monoids, the primely generated refinement monoids. Moreover any finitely generated conical refinement monoid arises as $M(E, C)$ for some adaptable separated graph $(E, C)$; see [4] for all these results, which we have also summarized in Section 1.

Having identified the suitable combinatorial object, namely adaptable separated graphs, we associate to each such separated graph a suitable inverse semigroup. Our guiding model is the graph inverse semigroup defined in [21], but difficulties arise in trying to modify the definition of the graph inverse semigroup from [21] to our context. These arise from the fact that the algebra relations naturally attached to a separated graph [7] are not tame; that is, they do not force commutation of range projections of the generating partial isometries, which precludes their generating an inverse semigroup. This problem was solved in [6] by forming a universal quotient, called the tame algebra of the separated graph, in which the range projections of the generating partial isometries do commute. Unfortunately, though, this introduced a new difficulty problem: in general, passing from the universal $C^{*}$-algebra or algebra of a separated graph to its tame quotient as in [6] induces a nontrivial map from 
the monoid $M(E, C)$ to the monoid $\mathcal{V O}(E, C)$ or $\mathcal{V} L_{K}^{\mathrm{ab}}(E, C)$; so we can no longer be sure that we are representing the desired monoid $M(E, C)$.

Our solution to this problem is to introduce a set of auxiliary variables $\left\{t_{i}^{v}: i \in \mathbb{N}, v \in\right.$ $\left.E^{0}\right\}$ which are designed to tame the natural relations associated to the separated graph $(E, C)$, without altering the associated monoid. Indeed the starting point of our paper is the construction of an inverse semigroup $S(E, C)$ for any adaptable separated graph $(E, C)$, which makes use of these auxiliary variables $t_{i}^{v}$. The crucial semilattice of idempotents $\mathcal{E}$ is described solely in terms of paths and monomials in the separated graph, and we are able to show that $S(E, C)$ is an $E^{*}$-unitary inverse semigroup, and consequently the tight groupoid $\mathcal{G}_{\text {tight }}(S(E, C))$ is an ample Hausdorff groupoid (see Section 2). Following the by-now well-trodden path described in [17], we then build the space of tight filters $\widehat{\mathcal{E}}_{\text {tight }}$ associated to $S(E, C)$, and we relate it to the space of infinite paths in our separated graph $(E, C)$. The desired groupoid is obtained as the groupoid $\mathcal{G}_{\text {tight }}(S(E, C))$ of germs of the canonical action of $S(E, C)$ on the space $\widehat{\mathcal{E}}_{\text {tight }}$ of tight filters. Our main result is Theorem 7.5: for any adaptable separated graph $(E, C)$, the type semigroup of the ample Hausdorff groupoid $\mathcal{G}_{\text {tight }}(S(E, C))$ is canonically isomorphic to the graph monoid $M(E, C)$. In particular, every finitely generated conical refinement monoid arises as the type semigroup of a groupoid in our class.

We show in addition that the groupoids $\mathcal{G}:=\mathcal{G}_{\text {tight }}(S(E, C)$ ) are all amenable (Proposition 6.2), and that the corresponding Steinberg algebras $A_{K}(\mathcal{G})$ and $C^{*}$-algebras $C^{*}(\mathcal{G})=$ $\mathcal{C}_{r}^{*}(\mathcal{G})$ can be described as universal objects for appropriate generators and relations (Theorem 4.14 and Corollary 4.15). We also obtain a very concrete description of the groupoid $\mathcal{G}_{\text {tight }}(S(E, C)$ ) (see Theorem 5.3), similar to the one given in [22] for usual graphs, which makes computations much more tractable.

We briefly outline the contents of this paper. In Section 1, we record the basic definitions and results we will need throughout the rest of the paper. In particular we introduce the key notion of an adaptable separated graph. In Section 2, we define a natural inverse semigroup $S(E, C)$ associated to an adaptable separated graph $(E, C)$. We provide two equivalent definitions of the semigroup and analyse its semilattice of idempotents $\mathcal{E}$. We also show that $S(E, C)$ is an $E^{*}$-unitary inverse semigroup (Proposition 2.19). We analyse the structure of the spaces of filters, ultrafilters, and tight filters on $\mathcal{E}$ in Section 3. We show in Theorem 3.6 that the space of ultrafilters on $\mathcal{E}$ coincides with the space of tight filters on $\mathcal{E}$. In Section 4, we show that the natural $K$-algebra $\mathcal{S}_{K}(E, C)$, associated to an adaptable separated graph $(E, C)$ is isomorphic to the Steinberg algebra $A_{K}\left(\mathcal{G}_{\text {tight }}(S(E, C))\right)$ of the groupoid $\mathcal{G}_{\text {tight }}(S(E, C))$ of germs of the canonical action of $S(E, C)$ on the space of tight filters on $\mathcal{E}$. In Section 5, we provide an equivalent, more concrete picture of $\mathcal{G}_{\text {tight }}(S(E, C))$. This is used in Section 6 to show that $\mathcal{G}_{\text {tight }}(S(E, C))$ is an amenable groupoid. We finish by showing in Section 7 that, for any adaptable separated graph $(E, C)$, the type $\operatorname{semigroup} \operatorname{Typ}\left(\mathcal{G}_{\text {tight }}(S(E, C))\right)$ of the groupoid $\mathcal{G}_{\text {tight }}(S(E, C))$ is naturally isomorphic to the monoid $M(E, C)$ associated to $(E, C)$. We deduce from the results of [4] that every finitely generated conical refinement monoid arises as the type semigroup of the groupoid associated to an adaptable separated graph. 


\section{Preliminaries.}

In this section, we will recall the basic definitions and results needed to follow the paper.

1.1. Basics on commutative monoids. We will denote by $\mathbb{N}$ the semigroup of positive integers, and by $\mathbb{Z}^{+}$the monoid of non-negative integers.

Given a commutative monoid $M$, we set $M^{*}:=M \backslash\{0\}$. We say that $M$ is conical if $M^{*}$ is a semigroup, that is, if, for all $x, y$ in $M, x+y=0$ only when $x=y=0$.

We say that $M$ is a refinement monoid if, for all $a, b, c, d$ in $M$ such that $a+b=c+d$, there exist $w, x, y, z$ in $M$ such that $a=w+x, b=y+z, c=w+y$ and $d=x+z$.

A basic example of a refinement monoid is the monoid $M(E)$ associated to a countable row-finite graph $E$ [8, Proposition 4.4].

If $x, y \in M$, we write $x \leq y$ if there exists $z \in M$ such that $x+z=y$. Note that $\leq$ is a translation-invariant pre-order on $M$, called the algebraic pre-order of $M$. All inequalities in commutative monoids will be with respect to this pre-order. An element $p$ in a monoid $M$ is a prime element if $p$ is not invertible in $M$, and, whenever $p \leq a+b$ for $a, b \in M$, then either $p \leq a$ or $p \leq b$. The monoid $M$ is primely generated if every non-invertible element of $M$ can be written as a sum of prime elements.

An element $x \in M$ is regular if $2 x \leq x$. An element $x \in M$ is an idempotent if $2 x=x$. An element $x \in M$ is free if $n x \leq m x$ implies $n \leq m$, for $n, m \in \mathbb{N}$. By [13, Theorem 4.5], any element of a primely generated refinement monoid is either free or regular.

1.2. Adaptable Separated Graphs. In [9] the first and third-named authors characterized the primely generated conical refinement monoids in terms of the so-called $I$-systems. Using this theory, the first, second and third-named authors obtained in [4] a combinatorial model for all finitely generated conical refinement monoids. The basic ingredient in this combinatorial description is the theory of separated graphs [7]. (Note that ordinary graphs are not sufficient for this purpose, see $[10,11]$.)

Definition 1.1 ([7, Definitions 2.1 and 4.1]). A separated graph is a pair $(E, C)$ where $E$ is a directed graph, $C=\bigsqcup_{v \in E^{0}} C_{v}$, and $C_{v}$ is a partition of $s^{-1}(v)$ (into pairwise disjoint nonempty subsets) for every vertex $v$. If $v$ is a sink, we take $C_{v}$ to be the empty family of subsets of $s^{-1}(v)$.

If all the sets in $C$ are finite, we shall say that $(E, C)$ is a finitely separated graph.

Given a finitely separated graph $(E, C)$, we define the monoid of the separated graph $(E, C)$ to be the commutative monoid given by generators and relations as follows:

$$
M(E, C)=\left\langle a_{v}\left(v \in E^{0}\right): a_{v}=\sum_{\{e \in X\}} a_{r(e)} \text { for every } X \in C_{v}, v \in E^{0}\right\rangle .
$$

We recall some basic graph-theoretic notions that we will need along the sequel. For further background, see, for example, [1].

Definition 1.2. Given a directed graph $E=\left(E^{0}, E^{1}, s, r\right)$ :

(1) We define a pre-order on $E^{0}$ (the path-way pre-order) by $v \leq w$ if and only if there is a directed path $\gamma$ in $E$ with $s(\gamma)=w$ and $r(\gamma)=v$.

(2) We will denote by $(I, \leq)$ the poset arising as the antisymetrization of $\left(E^{0}, \leq\right)$. Thus, denoting by $[v]$ the class of $v \in E^{0}$ in $I$, we have $[v] \leq[w]$ if and only if $v \leq w$. 
(3) We say that $E$ is transitive if every two vertices of $E^{0}$ are connected through a finite path.

Let us now define the main notion used throughout the paper.

Definition 1.3. Let $(E, C)$ be a finitely separated graph and let $(I, \leq)$ be the antisymmetrization of $\left(E^{0}, \leq\right)$. We say that $(E, C)$ is adaptable if $I$ is finite, and there exist a partition $I=I_{\text {free }} \sqcup I_{\text {reg }}$, and a family of subgraphs $\left\{E_{p}\right\}_{p \in I}$ of $E$ such that the following conditions are satisfied:

(1) $E^{0}=\bigsqcup_{p \in I} E_{p}^{0}$, where $E_{p}$ is a transitive row-finite graph if $p \in I_{\text {reg }}$ and $E_{p}^{0}=\left\{v^{p}\right\}$ is a single vertex if $p \in I_{\text {free }}$.

(2) For $p \in I_{\text {reg }}$ and $w \in E_{p}^{0}$, we have that $\left|C_{w}\right|=1$ and $\left|s_{E_{p}}^{-1}(w)\right| \geq 2$. Moreover, all edges departing from $w$ either belong to the graph $E_{p}$ or connect $w$ to a vertex $u \in E_{q}^{0}$, with $q<p$ in $I$.

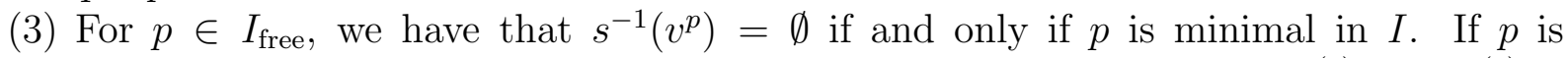
not minimal, then there is a positive integer $k(p)$ such that $C_{v^{p}}=\left\{X_{1}^{(p)}, \ldots, X_{k(p)}^{(p)}\right\}$. Moreover, each $X_{i}^{(p)}$ is of the form

$$
X_{i}^{(p)}=\{\alpha(p, i), \beta(p, i, 1), \beta(p, i, 2), \ldots, \beta(p, i, g(p, i))\},
$$

for some $g(p, i) \geq 1$, where $\alpha(p, i)$ is a loop, i.e., $s(\alpha(p, i))=r(\alpha(p, i))=v^{p}$, and $r(\beta(p, i, t)) \in E_{q}^{0}$ for $q<p$ in $I$. Finally, we have $E_{p}^{1}=\{\alpha(p, 1), \ldots, \alpha(p, k(p))\}$.

The edges connecting a vertex $v \in E_{p}^{0}$ to a vertex $w \in E_{q}^{0}$ with $q<p$ in $I$ will be called connectors.

This is the main theorem connecting graphs and monoids.

Theorem 1.4. [4] The following two statements hold:

(1) If $(E, C)$ is an adaptable separated graph, then $M(E, C)$ is a primely generated conical refinement monoid.

(2) For any finitely generated conical refinement monoid $M$, there exists an adaptable separated graph $(E, C)$ such that $M \cong M(E, C)$.

In particular, it is shown in [4] that, for an adaptable separated graph $(E, C)$, all the elements $a_{v}$, for $v \in E^{0}$, are prime elements of the monoid $M(E, C)$, and that $a_{v}$ is free (respectively, regular) in $M(E, C)$ if and only if $[v] \in I_{\text {free }}$ (respectively, $[v] \in I_{\text {reg }}$ ). We often refer to the elements of $I_{\text {free }}$ as free primes and to the elements of $I_{\text {reg }}$ as regular primes.

1.3. Groupoids and Steinberg algebras. Recall that a groupoid is a small category in which every morphism is an isomorphism (see [30] for further details). Given a groupoid $\mathcal{G}$, we will always denote its unit space by $\mathcal{G}^{(0)}$, the set of composable pairs by $\mathcal{G}^{(2)}$, and its source and range maps by $s$ and $r$, respectively. A bisection in $\mathcal{G}$ is a subset $U \subseteq \mathcal{G}$ such that the restrictions of $r$ and $s$ to $U$ are both injective.

A topological groupoid $\mathcal{G}$ is said to be étale if $\mathcal{G}^{(0)}$ is locally compact and Hausdorff in the relative topology, and its range map (equivalently, its source map) is a local homeomorphism from $\mathcal{G}$ to $\mathcal{G}^{(0)}$. It is easy to see that the topology of an étale groupoid admits a basis of open bisections. In an étale groupoid, $\mathcal{G}^{(0)}$ is open in $\mathcal{G}$. If, in addition, $\mathcal{G}$ is Hausdorff, then $\mathcal{G}^{(0)}$ is also closed in $\mathcal{G}$. An étale groupoid $\mathcal{G}$ is ample if $\mathcal{G}$ admits a basis of open compact bisections. 
Definition 1.5 ([34], [15]). Given an ample groupoid $\mathcal{G}$, and a field with involution $(K, *)$, the Steinberg algebra associated to $\mathcal{G}$ is defined to be the $*$-algebra over $K$

$$
A_{K}(\mathcal{G})=\operatorname{span}\left\{1_{B}: B \text { is an open compact bisection }\right\}
$$

with the convolution product

$$
(f g)(\gamma)=\sum_{\substack{\left(\gamma_{1}, \gamma_{2}\right) \in \mathcal{G}^{(2)} \\ \gamma_{1} \gamma_{2}=\gamma}} f\left(\gamma_{1}\right) g\left(\gamma_{2}\right)
$$

and the involution $f^{*}(\gamma)=f\left(\gamma^{-1}\right)^{*}$. When $\mathcal{G}$ is Hausdorff, $A_{K}(\mathcal{G})$ is just the $*$-algebra of compactly supported, locally constant functions $f: \mathcal{G} \rightarrow K$.

It is interesting to notice that $1_{B} 1_{D}=1_{B D}$, whenever $B$ and $D$ are compact open bisections in $\mathcal{G}$.

\section{The inVERSE SEMigroup ASSOCIATED TO AN ADAPTABLE SEPARATED GRAPH}

In this section we define the semigroup $S(E, C)$ associated to an adaptable separated graph $(E, C)$, and show that it is an $E^{*}$-unitary inverse semigroup.

We first define $S(E, C)$ as an abstract *-semigroup by using generators and relations, and then we prove that it is isomorphic to a concrete $*$-semigroup defined in terms of paths and monomials in our separated graph. This will be very useful in showing that $S(E, C)$ is an inverse semigroup and characterizing the semi-lattice of idempotents of $S(E, C)$, which is a key step for all what follows.

2.1. Definition of the inverse semigroup $S(E, C)$. We start by defining $S(E, C)$ by generators and relations. Roughly, it is generated by elements $v$ indexed by vertices of $E$, elements $e$ and $e^{*}$ indexed by edges of $E$, and some additional variables $t_{i}^{v}$ and $\left(t_{i}^{v}\right)^{-1}$ whose role is to "tame" the relations associated to the separated graph. Specifically, for each $v \in E^{0}$, we consider a collection of mutually commuting elements $\left\{t_{i}^{v},\left(t_{i}^{v}\right)^{-1} \mid i \in \mathbb{N}\right\}$ such that $t_{i}^{v}\left(t_{i}^{v}\right)^{-1}=v=\left(t_{i}^{v}\right)^{-1} t_{i}^{v}$, and $\left(t_{i}^{v}\right)^{*}=\left(t_{i}^{v}\right)^{-1}$. The way these elements interact with the natural generators corresponding to the separated graph is specified below. Before we give the definition of $S(E, C)$, let us fix some notation.

Notation. Assume notation provided in Definition 1.3. If $p \in I$ is non-minimal and free, we denote by $\sigma^{p}$ the map $\mathbb{N} \rightarrow \mathbb{N}$ given by

$$
\sigma^{p}(i)=i+k(p)-1
$$

Moreover, if $1 \leq j \leq k(p)$, we denote by $\sigma_{j}^{p}$ the unique bijective, non-decreasing map from $\{1, \ldots, k(p)\} \backslash\{j\}$ onto $\{1, \ldots, k(p)-1\}$.

We are now ready for the definition of the semigroup $S(E, C)$. Recall from Definition 1.3 the definition of an adaptable separated graph.

Definition 2.1. Given an adaptable separated graph $(E, C)$, denote by $S(E, C)$ the *semigroup (with 0) generated by $E^{0} \cup E^{1} \cup\left\{\left(t_{i}^{v}\right)^{ \pm} \mid i \in \mathbb{N}, v \in E^{0}\right\}$ and with defining

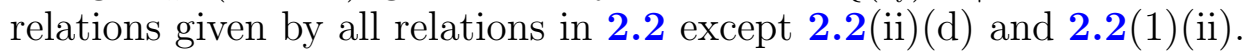


2.2 (Relations). There are two blocks of relations in which we are interested. In the first block we write the natural relations arising from the separated graph structure (cf. [7]). In the second block, we specify the relation between the generators of $S(E, C)$, using the special form of our adaptable separated graph.

\section{Block 1:}

(i) For all $v, w \in E^{0}$, we have $v \cdot w=\delta_{v, w} v$ and $v=v^{*}$.

(ii) For all $e \in E^{1}$, we have:

(a) $e=s(e) e=\operatorname{er}(e)$

(b) $e^{*} e=r(e)$

(c) $e^{*} f=\delta_{e, f} r(e)$ if $e, f \in X \subseteq C_{s(e)}$.

(d) $v=\sum_{e \in X} e e^{*}$, for $X \in C_{v}, v \in E^{0}$.

\section{Block 2:}

(1) For each free prime $p \in I$ and $i=1, \ldots, k(p)$, we have:

(i) $\quad \alpha(p, i)^{*} \alpha(p, i)=v^{p}$

$$
\alpha(p, i) \alpha(p, i)^{*}=v^{p}-\sum_{t=1}^{g(p, i)} \beta(p, i, t) \beta(p, i, t)^{*}
$$

(iii) For $i \neq j, \alpha(p, i) \alpha(p, j)=\alpha(p, j) \alpha(p, i)$, and $\alpha(p, i) \alpha(p, j)^{*}=\alpha(p, j)^{*} \alpha(p, i)$.

(iv) $\beta(p, i, s)^{*} \beta(p, j, t)=0$ if either $i \neq j$, or $i=j$ and $s \neq t$. (Note that when $i=j$ and $s \neq t$, these relations follow from the separated graph relations).

(v) $\alpha(p, i)^{*} \beta(p, i, t)=0=\beta(p, i, t)^{*} \alpha(p, i)$ for all $1 \leq i \leq k(p)$ and all $1 \leq t \leq g(p, i)$. Note that relations (i), (ii) and (v) follow from the separated graph relations.

(2) Moreover, in terms of the $\left\{t_{i}^{v}\right\}$, we impose the following relations:

(i) For each $v \in E^{0},\left\{\left(t_{i}^{v}\right)^{ \pm}: i \in \mathbb{N}\right\}$ is a family of mutually commuting elements such that

$$
v t_{i}^{v}=t_{i}^{v}=t_{i}^{v} v, \quad t_{i}^{v}\left(t_{i}^{v}\right)^{-1}=v=\left(t_{i}^{v}\right)^{-1} t_{i}^{v}, \quad\left(t_{i}^{v}\right)^{*}=\left(t_{i}^{v}\right)^{-1} .
$$

(ii) If $p \in I$ is regular, $e \in E^{1}$ is such that $s(e) \in E_{p}^{0}$ and $i \in \mathbb{N}$,

$$
t_{i}^{s(e)} e=e t_{i}^{r(e)} \text {. }
$$

(iii) If $p \in I$ is free, $i \in \mathbb{N}, 1 \leq j \leq k(p)$ and $1 \leq s \leq g(p, j)$,

$$
\left(t_{i}^{v^{p}}\right)^{ \pm} \beta(p, j, s)=\beta(p, j, s)\left(t_{\sigma^{p}(i)}^{r(\beta(p, j, s))}\right)^{ \pm},
$$

(iv) If $p \in I$ is free, $i \neq j$, and $1 \leq s \leq g(p, j)$,

$\alpha(p, i) \beta(p, j, s)=\beta(p, j, s) t_{\sigma_{j}^{p}(i)}^{r(\beta, j, s))}$, and $\alpha(p, i)^{*} \beta(p, j, s)=\beta(p, j, s)\left(t_{\sigma_{j}^{p}(i)}^{r(\beta(p, j, s))}\right)^{-1}$.

(v) If $p \in I$ is free, $t_{i}^{v^{p}} \alpha(p, j)=\alpha(p, j) t_{i}^{v^{p}}$ and $t_{i}^{v^{p}} \alpha(p, j)^{*}=\alpha(p, j)^{*} t_{i}^{v^{p}}$ for all $i \in \mathbb{N}$ and $j \in\{1, \ldots, k(p)\}$.

Remark 2.3. Since we are working within the category of $*$-semigroups, the $*$-relations of all the relations described in $\mathbf{2 . 2}$ are indeed enforced in the $*$-semigroup $S(E, C)$.

We next plan to provide a different description of $S(E, C)$. This will be given via the paths that one can intuitively associate to any adaptable separated graph. We show in 
Proposition 2.13 that $S(E, C)$ is isomorphic to this semigroup, which we will momentarily denote by $S$.

Let us start defining the notion of finite paths in this setting. Roughly, a finite path is described as follows: consider a sequence of elements $p_{1}>p_{2}>\ldots>p_{n}$ of the poset $I$, and for each $i$ a path $\gamma_{i}$ in $E_{p_{i}}$; we form a finite path by connecting the $\gamma_{i}$ together via the connectors $\beta$. Diagrammatically, we may write

$$
p_{1} \curvearrowright^{\beta_{1,2}} p_{2} \curvearrowright^{\beta_{2,3}} \ldots \curvearrowright^{\beta_{n-1, n}} p_{n} .
$$

More formally, we make the following definition.

Definition 2.4 (Finite paths). Let $(E, C)$ be an adaptable separated graph. Then, we define a step from a vertex $v \in E_{p}^{0}$ to a vertex $w \in E_{q}^{0}$ with $q<p$, denoted by $\hat{\gamma}_{v, w}$, as follows:

(1) if $v=v^{p}$ for $p$ a free prime, then a step from $v^{p}$ to $w$ is defined as

$$
\hat{\gamma}_{v, w}:=\alpha(p, i)^{m} \beta(p, i, t) \text { for some } i \text { and some } m \geq 0 \text {, where } r(\beta(p, i, t))=w \text {. }
$$

(2) if $v \in E_{p}^{0}$ for a regular prime $p$, then a step from $v$ to $w$ is defined as

$$
\hat{\gamma}_{v, w}:=\gamma \beta, \text { with } s(\beta)=v^{\prime}, r(\beta)=w,
$$

where $\gamma$ is a directed path of finite length connecting $v$ and $v^{\prime}$ in $E_{p}$, and $\beta$ is a connector from $v^{\prime}$ to $w$.

Then, given two vertices $v \in E_{p}^{0}$ and $w \in E_{q}^{0}$ with $p>q$ in $I$, we define a finite path from $v$ to $w$ as a concatenation of steps, i.e. we find $p=q_{0}>q_{1}>q_{2}>\ldots>q_{n}=q$, and vertices $v_{i} \in E_{q_{i}}^{0}$, with $v_{0}=v$ and $v_{n}=w$, such that

$$
\gamma_{v, w}:=\hat{\gamma}_{v_{0}, v_{1}} \ldots \hat{\gamma}_{v_{n-1}, v_{n}}
$$

We say that the path $\gamma_{v, w}$ has depth $n$, denoted by $\operatorname{depth}\left(\gamma_{v, w}\right)=n$.

A trivial finite path is a single vertex $v \in E^{0}$. These are the finite paths of depth 0 .

Given two finite paths $\gamma_{1}, \gamma_{2}$, we will write $\gamma_{1} \prec \gamma_{2}$ if $\gamma_{2}=\gamma_{1} \eta$ for some other finite path $\eta$; we write $\gamma_{1} \nprec \gamma_{2}$ otherwise.

Remark 2.5. Our finite paths are only some of the allowed paths of finite length in $E$. In particular note that this definition does not agree with the usual notion of a finite path in the underlying directed graph $E$. When we want to refer to the usual notion for a path in the graph $E$, we will speak of a path of finite length in the graph $E$. The usual length of such a path of finite length $\gamma$ will be denoted by $|\gamma|$. Later on, we will also give a technical sense to the following terms: infinite path, semifinite path, and $\mathcal{E}$-path.

Definition 2.6 (Monomials). We continue with our standing assumptions on $(E, C)$. We now define the monomials as the possible multiplicative expressions one can form using generators (excluding connectors) corresponding to a given prime. They will be denoted by $\mathbf{m}(p)$ for $p \in I$. Namely,

(1) if $p$ is a free prime, we define

$$
\mathbf{m}(p)=\left(t_{i_{1}}^{v^{p}}\right)^{d_{1}} \ldots\left(t_{i_{r}}^{v^{p}}\right)^{d_{r}} \prod_{j=1}^{k(p)} \alpha(p, j)^{k_{j}}\left(\alpha(p, j)^{*}\right)^{l_{j}}, d_{1}, \ldots, d_{r} \in \mathbb{Z} \backslash\{0\}, r \geq 0, k_{j}, l_{j} \geq 0
$$


(2) if $p$ is a regular prime, we define

$$
\mathbf{m}(p)=\left(t_{i_{1}}^{v}\right)^{d_{1}} \ldots\left(t_{i_{r}}^{v}\right)^{d_{r}} \gamma \nu^{*}
$$

where $\gamma, \nu$ are paths of finite length in $E_{p}$ satisfying $s(\gamma)=v, v \in E_{p}^{0}$, and $r(\gamma)=r(\nu)$.

For $p$ a free prime, two monomials $\mathbf{m}(p)$ and $\mathbf{m}^{\prime}(p)$ corresponding to $p$ are equal if they have the same exponents of $t_{i}^{v^{p}}, \alpha(p, j)$ and $\alpha(p, j)^{*}$. For $p$ a regular prime, two monomials $\mathbf{m}(p)$ and $\mathbf{m}^{\prime}(p)$ corresponding to $p$ are equal if they have the same exponents of $t_{i}^{v}$, and $\gamma=\gamma^{\prime}$, $\nu=\nu^{\prime}$, where $\gamma, \nu$ correspond to $\mathbf{m}(p)$ and $\gamma^{\prime}, \nu^{\prime}$ correspond to $\mathbf{m}^{\prime}(p)$.

Guided by the multiplication rules defined in $\mathbf{2 . 2}$, we will define an inverse semigroup $S$ using finite paths and monomials. For the moment, let us deal with the set of monomials at a given $p \in I$.

Lemma 2.7. For each $p \in I$, the set of monomials at $p$, together with $\{0\}$ in case $p \in I_{\text {reg }}$, forms a *-semigroup. Moreover we have

$$
\mathbf{m}(p) \mathbf{m}(p)^{*} \mathbf{m}(p)=\mathbf{m}(p)
$$

for each monomial $\mathbf{m}(p)$.

Proof. Assume first that $p$ is regular. Then, by using our assumption that $\left|s_{E_{p}}(v)\right| \geq 2$ for all $v \in E_{p}^{0}$ (Definition 1.3(2)), we see, using [1, Corollary 1.5.12], that the set of monomials $\mathbf{m}(p)$ at $p$, together with $\{0\}$, can be identified with a $*$-subsemigroup of the multiplicative semigroup of the Leavitt path algebra $L_{\mathbb{Z}\left[t_{i}^{ \pm}\right]}\left(E_{p}\right)$, where $\mathbb{Z}\left[t_{i}^{ \pm}\right]$is the ring of Laurent polynomials on $\left\{t_{i} \mid i \in \mathbb{N}\right\}$. Under this correspondence $\left(t_{i}^{v}\right)^{ \pm} \longleftrightarrow\left(t_{i}\right)^{ \pm} \cdot v$ for each $v \in E_{p}^{0}$. Equality (2.1) is easily verified.

Suppose now that $p$ is free. Then, the multiplication of $p$-monomials is defined using the rules in 2.2. Concretely, suppose that

$$
\mathbf{m}_{s}(p)=\prod_{i}\left(t_{i}^{v^{p}}\right)^{d_{i}^{(s)}} \prod_{j=1}^{k(p)} \alpha(p, j)^{k_{j}^{(s)}}\left(\alpha(p, j)^{*}\right)^{l_{j}^{(s)}}
$$

for $s=1,2,3$, then

$$
\mathbf{m}_{1}(p) \mathbf{m}_{2}(p)=\mathbf{m}_{3}(p)
$$

if and only if $d_{i}^{(3)}=d_{i}^{(1)}+d_{i}^{(2)}$ for all $i \in \mathbb{N}$ and $k_{j}^{(3)}=\max \left\{k_{j}^{(1)}, k_{j}^{(1)}+k_{j}^{(2)}-l_{j}^{(1)}\right\}$, and $l_{j}^{(3)}=\max \left\{l_{j}^{(2)}, l_{j}^{(2)}+l_{j}^{(1)}-k_{j}^{(2)}\right\}$ for each $j \in\{1, \ldots, k(p)\}$. Associativity, as well as the formula (2.1), are easily checked.

Using this whole data, we define the desired semigroup $S$. First, we fix the set:

Definition 2.8. Let $(E, C)$ be an adaptable separated graph. Then, we define $S$ to be the union of $\{0\}$ and the set of all triples $(\gamma, \mathbf{m}(p), \eta)$, where $\gamma, \eta$ are finite paths (Definition 2.4), $\mathbf{m}(p)$ is a monomial at some prime $p \in I$ (Definition 2.6), and $r(\gamma)=s(\mathbf{m}(p)), r(\eta)=$ $r(\mathbf{m}(p))$. So, $S$ consists of combinations of "finite paths" and monomials $\mathbf{m}$. We shall use a less formal notation, writing the elements of $S$ just as concatenations $\gamma \mathbf{m}(p) \eta^{*}$ of a finite path, a monomial and the star of a finite path. Note that finite paths do not involve the variables $t_{i}^{v}$, whilst monomials may involve these variables. 
Our next goal is to show that $S$ is a *-semigroup, obeying the multiplication rules stated in 2.2. It is important to highlight here that only the purely multiplicative relations in $\mathbf{2 . 2}$ are used to define the product of $S$. Explicitly, this means that relations 2.2(ii)(d) and 2.2(1)(ii) are not used to define it. In order to define its product, let us firstly define what we call the translation part $\phi_{\eta}(\mathbf{m}(p))$ for $\eta$ a finite path from $v \in E_{p}^{0}$ to $w \in E_{p^{\prime}}^{0}$, with $p>p^{\prime}$, and $\mathbf{m}(p)$ a monomial at $p$. This translation part is a monomial involving only the variables $\left(t_{i}^{r(\eta)}\right)^{ \pm}$, designed to satisfy the relation

$$
\mathbf{m}(p) \eta=\tilde{\eta} \phi_{\eta}(\mathbf{m}(p))
$$

for a suitable finite path $\tilde{\eta}$, in case the product $\mathbf{m}(p) \eta$ is nonzero.

Assume first that $p$ is a free prime. By definition, the monomial $\mathbf{m}(p)$ is of the form

$$
\mathbf{m}(p)=\left(t_{i_{1}}^{v^{p}}\right)^{d_{1}} \ldots\left(t_{i_{r}}^{v^{p}}\right)^{d_{r}} \prod_{j=1}^{k(p)} \alpha(p, j)^{k_{j}}\left(\alpha(p, j)^{*}\right)^{l_{j}}
$$

and the finite path to $w \in E_{p^{\prime}}^{0}$ has the form

$$
\eta=\hat{\gamma}_{v^{p}, v_{1}} \hat{\gamma}_{v_{1}, v_{2}} \ldots \hat{\gamma}_{v_{n}, w}=\alpha(p, i)^{m} \beta(p, i, t) \hat{\gamma}_{v_{1}, v_{2}} \ldots \hat{\gamma}_{v_{n}, w} \text { with } r(\beta(p, i, t))=v_{1} .
$$

We write $\eta=\eta_{1} \eta_{2}$, where $\eta_{1}=\alpha(p, i)^{m} \beta(p, i, t)$ and $\eta_{2}=\hat{\gamma}_{v_{1}, v_{2}} \ldots \hat{\gamma}_{v_{n}, w}$.

Thus, applying the commutation rules 2.2(1)(iii), we get

$$
\begin{gathered}
\mathbf{m}(p) \eta=\left(t_{i_{1}}^{v^{p}}\right)^{d_{1}} \ldots\left(t_{i_{r}}^{v^{p}}\right)^{d_{r}} \prod_{j=1}^{k(p)} \alpha(p, j)^{k_{j}}\left(\alpha(p, j)^{*}\right)^{l_{j}} \alpha(p, i)^{m} \beta(p, i, t) \eta_{2} \\
\left(t_{i_{1}}^{v^{p}}\right)^{d_{1}} \ldots\left(t_{i_{r}}^{v^{p}}\right)^{d_{r}}\left(\prod_{j \neq i} \alpha(p, j)^{k_{j}}\left(\alpha(p, j)^{*}\right)^{l_{j}}\right) \underbrace{\alpha(p, i)^{k_{i}}\left(\alpha(p, i)^{*}\right)^{l_{i}} \alpha(p, i)^{m} \beta(p, i, t)}_{(*)} \eta_{2} .
\end{gathered}
$$

Looking carefully at $(*)$, we just have two possibilities:

(1) if $l_{i}>m$, then $(*)=0$ by $2.2(1)(\mathrm{i}),(\mathrm{v})$; and

(2) if $l_{i} \leq m$, then $(*)=\alpha(p, i)^{k_{i}+m-l_{i}} \beta(p, i, t)$.

Hence, in the latter case (the other is zero), one has that

$$
\mathbf{m}(p) \eta=\alpha(p, i)^{k_{i}+m-l_{i}} \beta(p, i, t) \eta_{2} \phi_{\eta}(\mathbf{m}(p))
$$

where $\phi_{\eta}(\mathbf{m}(p))$ is a monomial in $\left(t_{i}^{w}\right)^{ \pm}$that comes from:

- passing $\left(t_{i_{1}}^{v^{p}}\right)^{d_{1}} \ldots\left(t_{i_{r}}^{v^{p}}\right)^{d_{r}}$ to the right through $\eta$ using repeatedly the rules in 2.2(2), and

- passing the product $\prod_{j \neq i} \alpha(p, j)^{k_{j}}\left(\alpha(p, j)^{*}\right)^{l_{j}}$ to the right through $\eta$ using again repeatedly the rules in $\mathbf{2 . 2}(2)$. (Note that $\mathbf{2 . 2}(2)$ (iv) is used here in an essential way.)

We have thus shown that the relations 2.2 force us to define the product $\mathbf{m}(p) \eta$ in the following way:

Definition 2.9. Assume that $p \in I$ is free, and adopt the above notation for $\mathbf{m}(p)$ and $\eta$. Then we define the product $\mathbf{m}(p) \eta$ to be nonzero if and only if $v^{p}=s(\eta)$ and $l_{i} \leq m$. In this case we set

$$
\mathbf{m}(p) \eta=\tilde{\eta} \phi_{\eta}(\mathbf{m}(p))
$$


where $\tilde{\eta}=\alpha(p, i)^{k_{i}+m-l_{i}} \beta(p, i, t) \eta_{2}$ and $\phi_{\eta}(\mathbf{m}(p))$ is a monomial involving only the variables $\left(t_{s}^{r(\eta)}\right)^{ \pm}, s \in \mathbb{N}$, as described above.

We now consider the case where $p$ is a regular prime.

Definition 2.10. Assume that $p \in I$ is regular, and let

$$
\mathbf{m}(p)=\left(t_{i_{1}}^{v}\right)^{d_{1}} \ldots\left(t_{i_{r}}^{v}\right)^{d_{r}} \gamma \nu^{*}
$$

be a monomial at $p$, with $s(\gamma)=v, s(\nu)=v^{\prime}$ and $v, v^{\prime} \in E_{p}^{0}$. Let $\eta$ be a finite path from $v^{\prime}$ to $w$ of the form

$$
\eta=\hat{\gamma}_{v^{\prime}, v_{1}} \hat{\gamma}_{v_{1}, v_{2}} \ldots \hat{\gamma}_{v_{n}, w}=\gamma^{\prime} \beta \hat{\gamma}_{v_{1}, v_{2}} \ldots \hat{\gamma}_{v_{n}, w}
$$

where $\gamma^{\prime}$ is a path in the graph $E_{p}$ connecting $v^{\prime}$ and $v^{\prime \prime}$, and $\beta$ is a connector with $s(\beta)=$ $v^{\prime \prime}$ and $r(\beta)=v_{1}$.

The product $\mathbf{m}(p) \eta$ is nonzero if and only if $\gamma^{\prime}=\nu \gamma^{\prime \prime}$, and then

$$
\mathbf{m}(p) \eta=\left(t_{i_{1}}^{v}\right)^{d_{1}} \ldots\left(t_{i_{r}}^{v}\right)^{d_{r}}\left(\gamma \gamma^{\prime \prime}\right) \beta \hat{\gamma}_{v_{1}, v_{2}} \ldots \hat{\gamma}_{v_{n}, w}=\tilde{\eta} \phi_{\eta}(\mathbf{m}(p)),
$$

where $\tilde{\eta}=\gamma \gamma^{\prime \prime} \beta \hat{\gamma}_{v_{1}, v_{2}} \ldots \hat{\gamma}_{v_{n}, w}$ and $\phi_{\eta}(\mathbf{m}(p))$ is a monomial in $\left\{\left(t_{i}^{w}\right)^{ \pm}\right\}$that comes from passing $\left(t_{i_{1}}^{v^{\prime \prime}}\right)^{d_{1}} \ldots\left(t_{i_{r}}^{v^{\prime \prime}}\right)^{d_{r}}$ to the right through $\beta \hat{\gamma}_{v_{1}, v_{2}} \ldots \hat{\gamma}_{v_{n}, w}$ by repeated use of the rules in 2.2(2).

We have thus established the formula (2.2) for any prime $p$ in $I$, whenever the product $\mathbf{m}(p) \eta$ is nonzero. Note that $\phi_{\eta}(\mathbf{m}(p))$ only depends on $\mathbf{m}(p)$ and on the connectors appearing in $\eta$. In particular, one has $\phi_{\eta}(\mathbf{m}(p))=\phi_{\tilde{\eta}}(\mathbf{m}(p))$. Note also that $\phi_{\eta}\left(\mathbf{m}(p)^{*}\right)=\phi_{\eta}(\mathbf{m}(p))^{*}$, so that $\phi_{\eta}$ preserves adjoints.

Using the above partial definitions, we are now ready to fully define the multiplication of the elements in $S$.

Definition 2.11. Let $\gamma_{1} \mathbf{m}(p) \eta_{1}^{*}$ and $\gamma_{2} \mathbf{n}\left(p^{\prime}\right) \eta_{2}^{*}$ be two elements in $S$. We define

$$
\gamma_{1} \mathbf{m}(p) \eta_{1}^{*} \cdot \gamma_{2} \mathbf{n}\left(p^{\prime}\right) \eta_{2}^{*}= \begin{cases}\gamma_{1}\left[\mathbf{m}(p) \phi_{\eta_{1}^{\prime}}\left(\mathbf{n}\left(p^{\prime}\right)\right)\right]\left(\eta_{2} \tilde{\eta}_{1}^{\prime}\right)^{*} & \text { if } \eta_{1}=\gamma_{2} \eta_{1}^{\prime} \text { and }\left(p^{\prime}\right)^{*} \eta_{1}^{\prime} \neq 0 \\ \gamma_{1} \tilde{\gamma}_{2}^{\prime}\left[\phi_{\gamma_{2}^{\prime}}(\mathbf{m}(p)) \mathbf{n}\left(p^{\prime}\right)\right] \eta_{2}^{*} & \text { if } \gamma_{2}=\eta_{1} \gamma_{2}^{\prime} \text { and } \mathbf{m}(p) \gamma_{2}^{\prime} \neq 0 \\ 0 & \text { otherwise }\end{cases}
$$

Lemma 2.12. Let $(E, C)$ be an adaptable separated graph. Then the set $S$, endowed with the above operation, is a *-semigroup, and we have $s=s s^{*} s$ for all $s \in S$.

Proof. The only delicate point is the associativity. Assume that we have three elements $s_{i}=\gamma_{i} \mathbf{m}\left(p_{i}\right) \eta_{i}^{*}$. Write $A=\left(s_{1} s_{2}\right) s_{3}$ and $B=s_{1}\left(s_{2} s_{3}\right)$. We will show that if $A \neq 0$ then $B \neq 0$ and $A=B$. The result then follows from symmetry.

So assume that $A \neq 0$. We will suppose that $p_{1}, p_{2}, p_{3}$ are free primes, leaving the easier case where one of them is regular to the reader. There are four cases to consider, all similar. So we will only provide the details in one case. Recall that associativity of the semigroup of monomials at a given prime has been established in Lemma 2.7.

Write

$$
\mathbf{m}\left(p_{s}\right)=\prod_{i}\left(t_{i}^{v^{p_{s}}}\right)^{d_{i}^{(s)}} \prod_{j=1}^{k\left(p_{s}\right)} \alpha\left(p_{s}, j\right)^{k_{j}^{(s)}}\left(\alpha\left(p_{s}, j\right)^{*}\right)^{l_{j}^{(s)}}
$$

for $s=1,2,3$. We suppose that $\eta_{1}=\gamma_{2} \eta_{1}^{\prime}$ and that $k_{i_{0}}^{(2)} \leq m_{1}$, where

$$
\eta_{1}^{\prime}=\alpha\left(p_{2}, i_{0}\right)^{m_{1}} \beta\left(p_{2}, i_{0}, t_{0}\right) \cdots \text {. }
$$


By Definition 2.11, we then have that $s_{1} s_{2}$ is nonzero and

$$
s_{1} s_{2}=\gamma_{1}\left[\mathbf{m}\left(p_{1}\right) \phi_{\eta_{1}^{\prime}}\left(\mathbf{m}\left(p_{2}\right)\right)\right]\left(\eta_{2} \tilde{\eta}_{1}^{\prime}\right)^{*},
$$

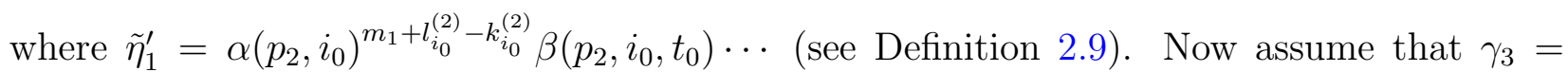
$\left(\eta_{2} \tilde{\eta}_{1}^{\prime}\right) \gamma_{3}^{\prime}$, and that $l_{i_{1}}^{(1)} \leq m_{2}$, where $\gamma_{3}^{\prime}=\alpha\left(p_{1}, i_{1}\right)^{m_{2}} \beta\left(p_{1}, i_{1}, t_{1}\right) \cdots$. Then the product $A=$ $\left(s_{1} s_{2}\right) s_{3}$ is nonzero and

$$
A=\left(\gamma_{1} \tilde{\gamma}_{3}^{\prime}\right)\left[\phi_{\gamma_{3}^{\prime}}\left(\mathbf{m}\left(p_{1}\right) \phi_{\eta_{1}^{\prime}}\left(\mathbf{m}\left(p_{2}\right)\right)\right) \mathbf{m}\left(p_{3}\right)\right] \eta_{3}^{*} .
$$

Let us check that $s_{2} s_{3} \neq 0$. First note that $\gamma_{3}=\eta_{2} \gamma_{3}^{\prime \prime}$, where $\gamma_{3}^{\prime \prime}=\tilde{\eta}_{1}^{\prime} \gamma_{3}^{\prime}$. Therefore, we have

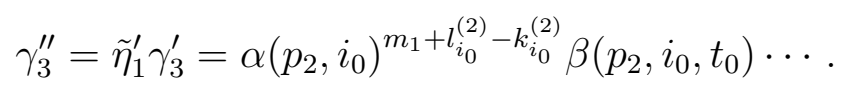

It follows that $s_{2} s_{3} \neq 0$ if and only if $l_{i_{0}}^{(2)} \leq m_{1}+l_{i_{0}}^{(2)}-k_{i_{0}}^{(2)}$, which is equivalent to our assumption $m_{1} \geq k_{i_{0}}^{(2)}$. Hence $s_{2} s_{3}$ is nonzero, and

$$
s_{2} s_{3}=\left(\gamma_{2} \tilde{\gamma}_{3}^{\prime \prime}\right)\left[\phi_{\gamma_{3}^{\prime \prime}}\left(\mathbf{m}\left(p_{2}\right)\right) \mathbf{m}\left(p_{3}\right)\right] \eta_{3}^{*},
$$

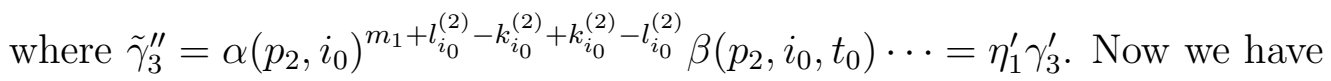

$$
\gamma_{2} \tilde{\gamma}_{3}^{\prime \prime}=\left(\gamma_{2} \eta_{1}^{\prime}\right) \gamma_{3}^{\prime}=\eta_{1} \gamma_{3}^{\prime}
$$

and so the product $s_{1}\left(s_{2} s_{3}\right)$ is nonzero if and only if $m_{2} \geq l_{i_{1}}^{(1)}$, which holds by our hypothesis. So we get

$$
B=s_{1}\left(s_{2} s_{3}\right)=\left(\gamma_{1} \tilde{\gamma}_{3}^{\prime}\right)\left[\phi_{\gamma_{3}^{\prime}}\left(\mathbf{m}\left(p_{1}\right)\right) \phi_{\gamma_{3}^{\prime \prime}}\left(\mathbf{m}\left(p_{2}\right)\right) \mathbf{m}\left(p_{3}\right)\right] \eta_{3}^{*}
$$

Hence, the equality $A=B$ follows from the computation

$$
\phi_{\gamma_{3}^{\prime}}\left(\mathbf{m}\left(p_{1}\right) \phi_{\eta_{1}^{\prime}}\left(\mathbf{m}\left(p_{2}\right)\right)\right)=\phi_{\gamma_{3}^{\prime}}\left(\mathbf{m}\left(p_{1}\right)\right) \phi_{\gamma_{3}^{\prime}} \phi_{\tilde{\eta}_{1}^{\prime}}\left(\mathbf{m}\left(p_{2}\right)\right)=\phi_{\gamma_{3}^{\prime}}\left(\mathbf{m}\left(p_{1}\right)\right) \phi_{\gamma_{3}^{\prime \prime}}\left(\mathbf{m}\left(p_{2}\right)\right)
$$

This concludes the proof of the result.

We have the following natural characterization of the *-semigoup $S$ :

Proposition 2.13. Let $(E, C)$ be an adaptable separated graph. Then, there is a natural *-isomorphism $S(E, C) \cong S$.

Proof. It is easy to see that there is a $*$-homomorphism $\varphi: S(E, C) \rightarrow S$ sending the generators of $S(E, C)$ to their canonical images in $S$. The obvious map $\psi: S \rightarrow S(E, C)$ is easily seen to be a $*$-homomorphism, which is clearly the inverse of $\varphi$.

2.2. Idempotents of $S(E, C)$. Another crucial point of the given characterization of $S(E, C)$ is the easy description of the idempotent elements in $S(E, C)$.

Definition 2.14 (Set of idempotents). We denote the set of idempotents in $S(E, C)$ by $\mathcal{E}$. By the rules of multiplication just defined, we easily deduce that the idempotents are the elements of the form:

$$
\gamma \mathbf{m}(p) \gamma^{*}
$$

where $\gamma$ is a finite path to $v \in E_{p}^{0}$ and the monomial $\mathbf{m}(p)$ is either equal to the product $\prod_{j=1}^{k(p)} \alpha(p, j)^{l_{j}}\left(\alpha(p, j)^{*}\right)^{l_{j}}$, when $p$ is free, or $\lambda \lambda^{*}$, for a path of finite length $\lambda$ in the graph $E_{p}$, with $s(\lambda)=v$, when $p$ is regular. Hence, an idempotent never contains the variables $t_{i}^{v}$. 
Remark 2.15. (1) Let $\gamma_{1} \mathbf{m}(p) \gamma_{1}^{*}$ and $\gamma_{2} \mathbf{n}\left(p^{\prime}\right) \gamma_{2}^{*}$ be two idempotents in $S(E, C)$. Notice that $\mathbf{m}(p) \mathbf{n}\left(p^{\prime}\right)=0$ if $p \neq p^{\prime}$. Moreover, if $p=p^{\prime}$ then $\mathbf{m}(p) \mathbf{n}\left(p^{\prime}\right)=\mathbf{n}\left(p^{\prime}\right) \mathbf{m}(p)$, i.e. idempotent monomials commute.

(2) Notice that if $e=\gamma \mathbf{m}(p) \gamma^{*}$ is an idempotent, then $\phi_{\eta}(\mathbf{m}(p))$ is trivial for any compatible finite path $\eta$, and moreover $\tilde{\eta}=\eta$ (see Definitions 2.9 and 2.10). In particular, when we multiply $e \cdot \gamma_{1} \mathbf{n}\left(p^{\prime}\right) \gamma_{2}^{*}$, with $\gamma_{1}=\gamma \eta$ and $\eta$ a non-trivial finite path, we have the following:

(a) If $p$ is free and $\mathbf{m}(p) \eta \neq 0$, then

$$
e \cdot \gamma_{1} \mathbf{n}\left(p^{\prime}\right) \gamma_{2}^{*}=\gamma \mathbf{m}(p) \gamma^{*} \gamma \eta \mathbf{n}\left(p^{\prime}\right) \gamma_{2}^{*}=\gamma \eta \mathbf{n}\left(p^{\prime}\right) \gamma_{2}^{*}=\gamma_{1} \mathbf{n}\left(p^{\prime}\right) \gamma_{2}^{*}
$$

Hence, it follows that either $e \cdot \gamma_{1} \mathbf{n}\left(p^{\prime}\right) \gamma_{2}^{*}=\gamma_{1} \mathbf{n}\left(p^{\prime}\right) \gamma_{2}^{*}$ or $e \cdot \gamma_{1} \mathbf{n}\left(p^{\prime}\right) \gamma_{2}^{*}=0$.

(b) If $p$ is regular, when computing $e \cdot \gamma_{1} \mathbf{n}\left(p^{\prime}\right) \gamma_{2}^{*}$, one has to look at the corresponding paths of finite length inside $E_{p}$. In this situation, depending on them, the product might be either zero or $\gamma_{1} \mathbf{n}\left(p^{\prime}\right) \gamma_{2}^{*}$, as before.

Gathering all the tools described, we finally conclude that $S$ is an inverse semigroup.

Proposition 2.16. Let $(E, C)$ be an adapated searated graph. Then, the semigroup $S(E, C)$ is an inverse semigroup.

Proof. By [23, Theorem 1.1.3], it is enough to show that given any two elements $e, f \in \mathcal{E}$, then one has that $e f=f e$. To this end, we write $e, f$ in standard form, i.e. $e=\gamma_{1} \mathbf{m}(p) \gamma_{1}^{*}$ and $f=\gamma_{2} \mathbf{n}\left(p^{\prime}\right) \gamma_{2}^{*}$, where $\gamma_{1}$ is a finite path to $p$ and $\gamma_{2}$ is a finite path to $p^{\prime}$.

Assume that $e f \neq 0$. Then either $\gamma_{1}=\gamma_{2}$ or $\gamma_{2}=\gamma_{1} \eta$ for a non-trivial finite path $\eta$ and the product $\mathbf{m}(p) \eta$ is nonzero, or $\gamma_{1}=\gamma_{2} \eta$ for a non-trivial finite path $\eta$ and $\mathbf{n}\left(p^{\prime}\right)^{*} \eta=\mathbf{n}\left(p^{\prime}\right) \eta$ is nonzero. In the first case we obtain $p=p^{\prime}$ and the result follows from the commutation of idempotent monomials. We shall see that in the second case we have $e f=f=f e$. By symmetry we will have $e f=e=f e$ in the third case.

So assume that $\gamma_{1}=\gamma_{2} \eta$ for a non-trivial finite path $\eta$ and that $\mathbf{m}(p) \eta \neq 0$. Then, using the computation in Remark 2.15 we get

$$
e f=\gamma_{1} \mathbf{m}(p) \gamma_{1}^{*} \gamma_{2} \mathbf{n}\left(p^{\prime}\right) \gamma_{2}^{*}=\gamma_{1} \eta \mathbf{n}\left(p^{\prime}\right) \gamma_{2}^{*}=f
$$

Similarly (or taking stars in the above), one gets $f e=f$.

Knowing that $S(E, C)$ is an inverse semigroup, it is natural to describe the natural order in $\mathcal{E}$, the set of projections. This will play an important role in the sequel. The following describes the order induced on $\mathcal{E}$. We omit the proof since it follows from the same techniques appearing in later proofs.

Lemma 2.17. Let $e, f \in \mathcal{E}$ described as $e=\gamma_{1} \mathbf{m}(p) \gamma_{1}^{*}, f=\gamma_{2} \mathbf{n}\left(p^{\prime}\right) \gamma_{2}^{*}$. Then ef $=0$ except in the following cases:

(1) $\gamma_{2}=\gamma_{1} \eta$ for some non-trivial finite path $\eta$ such that $\mathbf{m}(p) \eta \neq 0$. In this case, we have $f \leq e$.

(2) $\gamma_{1}=\gamma_{2} \eta$ for some non-trivial finite path $\eta$ such that $\mathbf{n}\left(p^{\prime}\right) \eta \neq 0$. In this case, we have $e \leq f$.

(3) $\gamma_{1}=\gamma_{2}$. In this case we have $p=p^{\prime}$, and 
(a) if $p$ is free, then ef $=\gamma_{1} \mathbf{m}^{\prime}(p) \gamma_{1}^{*}$, where, for each $1 \leq i \leq k(p)$, the exponent of $\alpha(p, i)$ in $\mathbf{m}^{\prime}(p)$ is the maximum between the exponents of $\alpha(p, i)$ in $\mathbf{m}(p)$ and in $\mathbf{n}(p)$.

(b) if $p$ is regular, and $\mathbf{m}(p)=\lambda \lambda^{*}, \mathbf{n}(p)=\mu \mu^{*}$, then either $\mu=\lambda \nu^{\prime}$, and then $f \leq e$, or $\lambda=\mu \lambda^{\prime}$, and then $e \leq f$.

Remark 2.18. If $p$ is a free prime, and $e=\gamma \mathbf{m}(p) \gamma^{*}, f=\gamma \mathbf{n}(p) \gamma^{*}$, we can also define the join $e \vee f$ of $e$ and $f$ by the formula

$$
e \vee f=\gamma \overline{\mathbf{m}}(p) \gamma^{*},
$$

where, for each $1 \leq i \leq k(p)$, the exponent of $\alpha(p, i)$ in $\overline{\mathbf{m}}(p)$ is the minimum amongst the exponents of $\alpha(p, i)$ in $\mathbf{m}(p)$ and in $\mathbf{n}(p)$. It is clear that $e \vee f$ is the least upper bound of $e$ and $f$ in $\mathcal{E}$.

2.3. The semigroup $S(E, C)$ is $E^{*}$-unitary. Continuing our analysis of the semigroup $S(E, C)$, in this last part of the section we show that it is $E^{*}$-unitary; recall that an inverse semigroup with zero $S$ is $E^{*}$-unitary if for any $s \in S, e \in \mathcal{E} \backslash\{0\}, e \leq s$ implies $s \in \mathcal{E}$. This will have crucial implications later.

Proposition 2.19. Let $(E, C)$ be an adaptable separated graph. Then the associated inverse semigroup $S(E, C)$ is $E^{*}$-unitary.

Proof. Fix a nonzero idempotent $e=\gamma_{1} \mathbf{m}(p) \gamma_{1}^{*}$ and an element $s=\gamma_{2} \mathbf{n}(q) \gamma_{3}^{*}$, and suppose that $e \leq s$; that is, $e=e s$. We will distinguish several cases.

- $\left(\gamma_{1} \prec \gamma_{2}\right)$. By assumption, we have $\gamma_{2}=\gamma_{1} \gamma$, for some non-trivial finite path $\gamma$; hence,

$$
\gamma_{1} \mathbf{m}(p) \gamma_{1}^{*}=\gamma_{1} \mathbf{m}(p) \gamma_{1}^{*} \gamma_{2} \mathbf{n}(q) \gamma_{3}^{*}=\gamma_{1} \tilde{\gamma}\left[\phi_{\gamma}(\mathbf{m}(p)) \mathbf{n}(q)\right] \gamma_{3}^{*}
$$

Since $e$ is an idempotent, this is equal to $\gamma_{2} \mathbf{n}(q) \gamma_{3}^{*}$. Therefore, $\gamma_{1}=\gamma_{2}=\gamma_{3}$, contradicting $\gamma_{1} \prec \gamma_{2}$.

- $\left(\gamma_{1}=\gamma_{2}\right)$. In this case, we have

$$
\gamma_{1} \mathbf{m}(p) \gamma_{1}^{*}=\gamma_{1} \mathbf{m}(p) \mathbf{n}(q) \gamma_{3}^{*},
$$

obtaining that $\gamma_{1}=\gamma_{2}=\gamma_{3}, p=q$ and $\mathbf{m}=\mathbf{m n}$. We consider two cases:

(i) If $p \in I$ is free, then by the description of the monomials in this case, one obtains that $\mathbf{n}$ is an idempotent monomial. Therefore, $s$ is an idempotent, as desired.

(ii) If $p \in I$ is regular, then by the description of the monomials, it follows that $\mathbf{m}(p)=\alpha_{1} \alpha_{1}^{*}$ and $\mathbf{n}(q)=\alpha_{2} \alpha_{3}^{*}$ for some paths $\alpha_{1}, \alpha_{2}, \alpha_{3}$ in $E_{p}$. Hence,

$$
\alpha_{1} \alpha_{1}^{*}=\alpha_{1} \alpha_{1}^{*} \alpha_{2} \alpha_{3}^{*}
$$

which implies that $\alpha_{3}=\alpha_{2}$; hence, $s$ is an idempotent.

- $\left(\gamma_{2} \prec \gamma_{1}\right)$. In this case, let us write $\gamma_{1}=\gamma_{2} \eta$ for some non-trivial finite path $\eta$. We have

$$
\gamma_{1} \mathbf{m}(p) \gamma_{1}^{*}=\gamma_{1} \mathbf{m}(p) \eta^{*} \mathbf{n}(q) \gamma_{3}^{*}=\gamma_{1}\left[\mathbf{m}(p) \phi_{\eta}(\mathbf{n}(q))\right]\left(\gamma_{3} \tilde{\eta}\right)^{*}
$$

We get $\gamma_{1}=\gamma_{2} \eta=\gamma_{3} \tilde{\eta}$. Since $\operatorname{depth}(\tilde{\eta})=\operatorname{depth}(\eta)$, we have that $\operatorname{depth}\left(\gamma_{3}\right)=$ $\operatorname{depth}\left(\gamma_{2}\right)$, and consequently we obtain $\gamma_{2}=\gamma_{3}$ and $\tilde{\eta}=\eta$. Moreover, $\phi_{\eta}(\mathbf{n}(q))=1$. Now, we separate two cases: 
(i) If $q \in I$ is free, then, writing $\eta=\alpha(p, i)^{m} \beta(p, i, s) \cdots$, one gets from $\eta=\tilde{\eta}$ that the exponents of $\alpha(p, i)$ and of $\alpha(p, i)^{*}$ in $\mathbf{n}(q)$ are equal. Now, since $\phi_{\eta}(\mathbf{n}(q))=1$, we obtain that, for $j \neq i$, also the exponents of $\alpha(p, j)$ and $\alpha(p, j)^{*}$ in $\mathbf{n}(q)$ are equal, and $\mathbf{n}(q)$ does not involve the variables $t_{i}^{v^{q}}$. Hence, $\mathbf{n}(q)$ is an idempotent monomial. Consequently, $s=\gamma_{2} \mathbf{n}(q) \gamma_{2}^{*} \in \mathcal{E}$.

(ii) If $q \in I$ is regular, then $\mathbf{n}(q)=\alpha \mu^{*}$. Write $\eta=\alpha \mu^{\prime} \beta \cdots$, where $\beta$ is the first connector appearing in the expression of $\eta$. Recalling that $\mathbf{n}(q)^{*} \eta=\tilde{\eta} \phi_{\eta}\left(\mathbf{n}(q)^{*}\right)=$ $\eta$, we obtain

$$
\mu \mu^{\prime} \beta \cdots=\left(\mu \alpha^{*}\right)\left(\alpha \mu^{\prime} \beta \cdots\right)=\alpha \mu^{\prime} \beta \cdots,
$$

so that $\mu=\alpha$ and $\mathbf{n}(q)=\alpha \alpha^{*}$ is an idempotent monomial, showing that $s$ is idempotent.

As a consequence of Proposition 2.19 and [17, Proposition 6.4 and 6.2], we obtain the following result about the Hausdorff property of the tight groupoid $\mathcal{G}_{\text {tight }}(S(E, C))$ associated to the inverse semigroup $S(E, C)$ (see Section 4 for further details).

Corollary 2.20. Suppose that $(E, C)$ is an adapated separated graph. Then the tight groupoid $\mathcal{G}_{\text {tight }}(S(E, C))$ associated to $S(E, C)$ is Hausdorff.

\section{Filters of the inverse semigroup $S(E, C)$}

In this section we study the filters associated to the inverse semigroup $S(E, C)$, and we characterize its tight filters. They will be useful in the next section to determine the groupoid induced by the partial action of the inverse semigroup on the set of tight filters.

We first recall the definition of a filter in a semilattice $\mathcal{E}$ of idempotents.

Definition 3.1. A filter on $\mathcal{E}$ is a non-empty subset $\eta \subset \mathcal{E}$ such that :

(1) $0 \notin \eta$.

(2) If $e \in \eta$, and $f \in \mathcal{E}$ satisfy $e \leq f$, then $f \in \eta$.

(3) If $e, f \in \eta$, then ef $\in \eta$.

We will denote the set of filters on $\mathcal{E}$ as $\hat{\mathcal{E}}_{0}$.

From now on, and in accordance with the notation established in Section 2, we let $\mathcal{E}$ denote the semilattice of idempotents of the inverse semigroup $S(E, C)$ associated to a fixed adaptable separated graph $(E, C)$.

Recall that we can endow the set $\hat{\mathcal{E}}_{0}$ of filters with a natural topology such that it becomes a totally disconnected locally compact Hausdorff space. A basis of this topology is given as follows. For finite sets $X, Y \subseteq \mathcal{E}$, define

$$
\mathcal{U}(X, Y)=\left\{\eta \in \hat{\mathcal{E}}_{0} \mid X \subseteq \eta \text { and } Y \cap \eta=\emptyset\right\} .
$$

Then, $\{\mathcal{U}(X, Y) \mid X, Y \subseteq \mathcal{E}$ are finite $\}$ is a basis for the abovementioned topology.

Let us provide a concrete description of any $\eta \in \hat{\mathcal{E}}_{0}$ using the concrete form of the idempotent elements. Recall that an idempotent $e \in \mathcal{E}$ is of the form $\gamma \mathbf{m}(p) \gamma^{*}$ (see Definition 2.14), and that $\operatorname{depth}(\gamma)$ denotes the depth of the finite path $\gamma$. Also, remembering that the poset $I$ is finite, we see that the depth of any possible finite path $\gamma$ is bounded on $\mathbb{N}$.

In order to understand the filters, it is convenient to introduce the following definition. 
Definition 3.2. Let $\gamma$ be a finite path. A semifinite path $\mu$ starting at $\gamma$ is one of the following:

(1) If $r(\gamma)=v^{p}$, with $p$ a free prime, then

$$
\mu=\gamma \prod_{j=1}^{k(p)} \alpha(p, j)^{k_{j}}
$$

where $0 \leq k_{j} \leq \infty$ for all $j \in\{1, \ldots, k(p)\}$. We say that $\mu$ is an infinite path if $k_{j}=\infty$ for all $j \in\{1, \ldots, k(p)\}$.

(2) If $r(\gamma)=v$ with $v \in E_{p}^{0}$ and $p$ a regular prime, then

$$
\mu=\gamma \lambda
$$

where $\lambda$ is either a finite or an infinite path in the graph $E_{p}$. We say that $\mu$ is an infinite path if $\lambda$ is an infinite path in $E_{p}$.

Definition 3.3. An initial segment of the semifinite path $\mu$ is a semifinite path $\mu^{\prime}$ of the form $\mu^{\prime}=\gamma^{\prime} \lambda^{\prime}$, where $\gamma^{\prime}$ is a finite path such that $\gamma=\gamma^{\prime} \gamma^{\prime \prime}$ for some finite path $\gamma^{\prime \prime}$, and either:

(1) $\gamma=\gamma^{\prime}$, and

(i) if $p$ is free, then $\lambda^{\prime}=\prod_{j=1}^{k(p)} \alpha(p, j)^{l_{j}}$ with $l_{j} \in \mathbb{Z}^{+}$and $l_{j} \leq k_{j}$ for all $j=$ $1, \ldots, k(p)$, and

(ii) if $p$ is regular, then $\lambda^{\prime}$ is an initial segment of the path $\lambda$ in the graph $E_{p}$; or

(2) $\gamma^{\prime} \neq \gamma$, and

(i) if $\gamma^{\prime \prime}=\alpha(q, i)^{m} \beta(q, i, s) \cdots$, where $q \in I_{\text {free, }}$, then $\lambda^{\prime}=\prod_{j=1}^{k(q)} \alpha(q, j)^{t_{j}}$ with $t_{j} \in$ $\mathbb{Z}^{+}, j=1, \ldots, k(q)$, and $t_{i} \leq m$, and

(ii) if $\left[r\left(\gamma^{\prime}\right)\right] \in I_{\mathrm{reg}}$, then $\lambda^{\prime}$ is an initial segment of $\gamma^{\prime \prime}$ that does not contain a connector.

With these definitions of finite and semifinite paths, all finite paths are semifinite. However, there are semifinite paths of finite length which are not finite paths according to our definition.

Our goal is to show that the filters on $\mathcal{E}$ correspond to the collection of all semifinite paths. To this end, we need the following definition. Let $\mu=\gamma \lambda$ be a semifinite path. Then, for each initial segment $\mu^{\prime}=\gamma^{\prime} \lambda^{\prime}$ of $\mu$, we will denote the idempotent arising from $\mu^{\prime}$ as:

$$
e\left(\mu^{\prime}\right):=\gamma^{\prime} \lambda^{\prime}\left(\lambda^{\prime}\right)^{*}\left(\gamma^{\prime}\right)^{*} \in \mathcal{E} .
$$

Using this notion, we show the desired equivalence.

Theorem 3.4. Let $\mathcal{S}$ be the collection of all semifinite paths. Then there is a bijective correspondence

$$
\varphi: \mathcal{S} \rightarrow \hat{\mathcal{E}}_{0}
$$

such that, for $\mu \in \mathcal{S}$,

$$
\varphi(\mu)=\left\{e\left(\mu^{\prime}\right) \mid \mu^{\prime} \text { is an initial segment of } \mu\right\} .
$$

Proof. It is easy to see that $\varphi(\mu)$ is a filter, and that the map $\varphi$ is one-to-one.

It remains to check that $\varphi$ is surjective. Let $\eta$ be a filter on $\mathcal{E}$. Observe that, since $I$ is a finite poset, there is a maximum for the depths of the finite paths appearing in the expressions of the elements of $\eta$. Let $e=\gamma \mathbf{m}(p) \gamma^{*}$ be an element in $\eta$ such that $\gamma$ has maximum depth, 
say $k$. We distinguish two cases. If $p$ is a regular prime, then there exists a path (of finite or infinite length) $\lambda$ in $E_{p}$ such that

$$
\left\{\lambda^{\prime} \mid \gamma \lambda^{\prime}\left(\lambda^{\prime}\right)^{*} \gamma^{*} \in \eta\right\}=\left\{\lambda^{\prime} \mid \lambda=\lambda^{\prime} \lambda^{\prime \prime}\right\}
$$

that is, the set of paths $\lambda^{\prime}$ of finite length in $E_{p}$ such that $\gamma \lambda^{\prime}\left(\lambda^{\prime}\right)^{*} \gamma^{*}$ belongs to $\eta$ is the set of initial segments of the path $\lambda$. Write $\mu=\gamma \lambda$ for this path $\lambda$, and observe that $\varphi(\mu)=\eta$. If $p$ is a free prime, then for each $1 \leq j \leq k(p)$, we let $k_{j} \in \mathbb{Z}^{+} \cup\{\infty\}$ be the supremum of the positive integers $l_{j}$ such that $\eta$ contains an element of the form $\gamma \mathbf{m}^{\prime}(p) \gamma^{*}$ such that the exponent of $\alpha(p, j)$ in $\mathbf{m}^{\prime}(p)$ is $l_{j}$. Now set

$$
\mu=\gamma \prod_{j=1}^{k(p)} \alpha(p, j)^{k_{j}}
$$

which is a semifinite path.

We claim that $\varphi(\mu)=\eta$. We first check that $\varphi(\mu) \subseteq \eta$. Write $\mu=\gamma \lambda$ and let $\mu^{\prime}=\gamma^{\prime} \lambda^{\prime}$ be an initial segment of $\mu$. If $r\left(\gamma^{\prime}\right)$ is a regular prime, then it follows easily that $\gamma^{\prime}\left(\lambda^{\prime}\right)\left(\lambda^{\prime}\right)^{*}\left(\gamma^{\prime}\right)^{*} \in \eta$, so that $e\left(\mu^{\prime}\right) \in \eta$. Assume that $q:=r\left(\gamma^{\prime}\right)$ is a free prime. If $\gamma^{\prime}=\gamma$, then write $\mu^{\prime}=\gamma \lambda^{\prime}$, where $\lambda^{\prime}=\prod_{j=1}^{k(p)} \alpha(p, j)^{l_{j}}\left(\alpha(p, j)^{*}\right)^{l_{j}}$, where the $l_{j}$ are non-negative integers with $l_{j} \leq k_{j}$ for all $j$. By definition of $k_{j}$, for each $1 \leq j \leq k(p)$ there exists $f_{j}=\gamma \mathbf{m}_{j}(p) \gamma^{*} \in \eta$ such that the exponent of $\alpha(p, j)$ in $\mathbf{m}_{j}(p)$ is at least $l_{j}$. Let $f=\prod_{j=1}^{k(p)} f_{j}$. Since $\eta$ is a filter, we have that $f \in \eta$, and the exponent of each $\alpha(p, j)$ in $f$ is $\geq l_{j}$, for $j=1, \ldots, k(p)$. Hence

$$
e\left(\mu^{\prime}\right) f=\left(\gamma \lambda^{\prime}\left(\lambda^{\prime}\right)^{*} \gamma^{*}\right) f=f .
$$

Since $\eta$ is a filter, we get that $e\left(\mu^{\prime}\right)=\gamma \lambda^{\prime}\left(\lambda^{\prime}\right)^{*} \gamma^{*} \in \eta$, as desired. Finally, assume now that $\gamma^{\prime} \neq \gamma$, and let

$$
\lambda^{\prime}=\prod_{j=1}^{k(q)} \alpha(q, j)^{t_{j}}\left(\alpha(q, j)^{*}\right)^{t_{j}}, \quad \gamma^{\prime \prime}=\alpha(q, i)^{m} \beta(q, i, s) \cdots,
$$

where $\gamma=\gamma^{\prime} \gamma^{\prime \prime}$. Since $t_{i} \leq m$, we have that $e\left(\mu^{\prime}\right) e=e$ (see Lemma 2.17). Therefore $e<e\left(\mu^{\prime}\right)$ and since $e \in \eta$ and $\eta$ is a filter, we get that $e\left(\mu^{\prime}\right) \in \eta$. Hence we have verified that $\varphi(\mu) \subseteq \eta$.

Now we prove that $\eta \subseteq \varphi(\mu)$. If $f=\gamma^{\prime} \mathbf{n}(q)\left(\gamma^{\prime}\right)^{*}$ is another element of $\eta$, then the condition that ef $\neq 0$, Lemma 2.17, and the maximality of $k$, give that $\gamma=\gamma^{\prime} \gamma^{\prime \prime}$ for a finite path $\gamma^{\prime \prime}$. If $\gamma^{\prime \prime}$ is trivial, then $\gamma=\gamma^{\prime}$, and by definition of $\mu$ we have that $\mathbf{n}(p)=\lambda^{\prime}\left(\lambda^{\prime}\right)^{*}$, where $\lambda^{\prime}$ is an initial segment of $\lambda$ in the graph $E_{p}$ if $p$ is regular, and $\lambda^{\prime}=\prod_{j=1}^{k(p)} \alpha(p, j)^{l_{j}}$, where $l_{j}$ are non-negative integers with $l_{j} \leq k_{j}$ if $p$ is free. In either case, $\mu^{\prime}=\gamma \lambda^{\prime}$ is an initial segment of $\mu$ and so, that $f \in \varphi(\mu)$. Assume that $\gamma^{\prime} \neq \gamma$. If $q$ is regular, then $f=\gamma^{\prime} \lambda^{\prime}\left(\lambda^{\prime}\right)^{*}\left(\gamma^{\prime}\right)^{*}$, where $\lambda^{\prime}$ is a path in $E_{q}$ and, since $f e \neq 0$, we get that $\lambda^{\prime}$ must be an initial segment of $\gamma^{\prime \prime}$. If $q$ is free, write $\gamma^{\prime \prime}=\alpha(q, i)^{m} \beta(q, i, s) \cdots$ and $\mathbf{n}(q)=\prod_{j=1}^{k(q)} \alpha(q, j)^{t_{j}}\left(\alpha(q, j)^{*}\right)^{t_{j}}$. Since $f e \neq 0$ we see from Lemma 2.17 that $t_{i} \leq m$, and thus $\mu^{\prime}:=\gamma \prod_{j=1}^{k(q)} \alpha(q, j)^{t_{j}}$ is an initial segment of $\mu$. Hence

$$
f=e\left(\mu^{\prime}\right) \in \varphi(\mu) .
$$

Corollary 3.5. The above isomorphism $\varphi$ restricts to a bijection between the set of infinite paths and the set $\hat{\mathcal{E}}_{\infty}$ of ultrafilters. 
Proof. This follows from Theorem 3.4 and the fact that the ultrafilters are precisely the maximal filters.

We can now show that the ultrafilters coincide with the tight filters. Recall that, by [17, Theorem 12.9], the space $\widehat{\mathcal{E}}_{\text {tight }}$ of tight filters coincides with the closure of the space of ultrafilters $\widehat{\mathcal{E}}_{\infty}$ in the space of filters $\widehat{\mathcal{E}}_{0}$.

Theorem 3.6. The space $\hat{\mathcal{E}}_{\infty}$ of ultrafilters is closed in the space $\hat{\mathcal{E}}_{0}$ of filters. Consequently, the space of ultrafilters coincides with the space of tight filters.

Proof. It is enough to show that the complement of $\hat{\mathcal{E}}_{\infty}$ in $\hat{\mathcal{E}}_{0}$ is open. Let $\eta=\varphi(\mu)$, where $\mu=\gamma \lambda$ is a semifinite path which is not an infinite path. If $w:=r(\gamma)$, with $w \in E_{p}^{0}$ and $p$ a regular prime, then $\lambda$ must be a finite path in the graph $E_{p}$. Let $X=\left\{\mu \mu^{*}\right\}$ and $Y=\left\{\mu e e^{*} \mu^{*} \mid e \in s_{E}^{-1}\left(r_{E}(\lambda)\right)\right\}$. Since $E$ is a row-finite graph, it follows that $Y$ is a finite set. Now

$$
\mathcal{U}(X, Y)=\{\eta\}
$$

which implies that $\eta$ is an isolated point of $\hat{\mathcal{E}}_{0}$ in this case.

Now suppose that $p$ is a free prime, and that $w=v^{p}$. Then, $\lambda=\prod_{j=1}^{k(p)} \alpha(p, j)^{k_{j}}$, and, by assumptions, there exists an index $i_{0}$ such that $k_{i_{0}}$ is finite. Assume, for convenience, that $i_{0}=1$. Define semifinite paths $\mu^{\prime}$ and $\delta$ by

$$
\mu^{\prime}:=\gamma \alpha(p, 1)^{k_{1}} \quad \text { and } \quad \delta:=\gamma \alpha(p, 1)^{k_{1}+1}
$$

which define idempotents $e\left(\mu^{\prime}\right)=\mu^{\prime}\left(\mu^{\prime}\right)^{*}, e(\delta)=\delta \delta^{*} \in \mathcal{E}$. Let

$$
Y_{1}=\left\{e\left(\mu^{\prime} \beta(p, 1, s)\right) \mid 1 \leq s \leq g(p, 1)\right\},
$$

and define

$$
X=\left\{e\left(\mu^{\prime}\right)\right\} \quad \text { and } \quad Y=\{e(\delta)\} \cup Y_{1} .
$$

Then $\mathcal{U}(X, Y)$ is a neighborhood of $\eta$ consisting entirely of semifinite paths which are not infinite. Indeed, if $\eta^{\prime} \in \mathcal{U}(X, Y)$, then either $\eta^{\prime}$ is of the form $\varphi\left(\mu^{\prime \prime}\right)$, where $\mu^{\prime \prime}$ is a semifinite path of the form

$$
\gamma \prod_{j=1}^{k(p)} \alpha(p, j)^{l_{j}}
$$

where $l_{1}=k_{1}$, which is therefore not an infinite path, or it is of the form $\varphi(\rho)$, where $\rho=\gamma \alpha(p, j)^{m} \beta(p, j, s) \rho^{\prime}$, where $j>1$ and $\rho^{\prime}$ is a semifinite path. Notice that in this case,

$$
e(\delta)=\gamma \alpha(p, 1)^{k_{1}+1}\left(\alpha(p, 1)^{*}\right)^{k_{1}+1} \gamma^{*} \in \varphi(\rho)=\eta^{\prime}
$$

because $e(\delta) \geq g$, where $g=e\left(\gamma \alpha(p, j)^{m} \beta(p, j, s)\right) \in \eta^{\prime}$. Hence, $e(\delta) \in \eta^{\prime}$, contradicting that $\eta^{\prime} \in \mathcal{U}(X, Y)$.

So, this second case is not possible and we obtain that $\mathcal{U}(X, Y)$ is a neighborhood of $\eta$ consisting entirely of semifinite paths which are not infinite.

We close this section by providing an important characterization of the topology associated to the space $\hat{\mathcal{E}}_{\infty}$. To do so, we use the identification of the space of ultrafilters $\hat{\mathcal{E}}_{\infty}$ with the space of infinite paths. 
Definition 3.7. We denote by $\mathcal{P}$ the set of semifinite paths of the form $\mu=\gamma \lambda$, where $\gamma$ is a finite path, and $\lambda$ is a path of finite length in the component of a regular prime, or $\lambda=\prod_{j=1}^{k(p)} \alpha(p, j)^{k_{j}}$ for $k_{j} \in \mathbb{Z}^{+}, 1 \leq j \leq k(p)$ for a free prime $p$.

Notice that every $e \in \mathcal{E}$ is of the form $e(\mu)$ for a unique $\mu \in \mathcal{P}$. Accordingly, elements of $\mathcal{P}$ will be called $\mathcal{E}$-paths. For $\mu \in \mathcal{P}$, write

$$
\mathcal{Z}(\mu)=\left\{\eta \in \hat{\mathcal{E}}_{\infty} \mid \mu \mu^{*} \in \eta\right\}
$$

Depending on the situation, $\mathcal{Z}(\mu)$ might also be denoted by the idempotent it determines, i.e., $\mathcal{Z}(e(\mu))$. Notice that $\mathcal{Z}(\mu)=\mathcal{U}\left(\left\{\mu \mu^{*}\right\}, \emptyset\right) \cap \hat{\mathcal{E}}_{\infty}$.

Given finite $X, Y \subseteq \mathcal{P}$, we write $\mathcal{U}^{\infty}(X, Y):=\mathcal{U}(X, Y) \cap \hat{\mathcal{E}}_{\infty}$. Then the induced topology on $\hat{\mathcal{E}}_{\infty}$ is generated by $\left\{\mathcal{U}^{\infty}(X, Y): X, Y \subseteq \mathcal{P}\right.$ are finite $\}$. The next result provides some properties of the sets $\mathcal{Z}(\mu)$ just defined.

Proposition 3.8. Let $\mathcal{P}$ be the set of $\mathcal{E}$-paths. Then

(1) the family $\{\mathcal{Z}(\mu) \mid \mu \in \mathcal{P}\} \cup\{\emptyset\}$ is closed under finite intersections;

(2) the family $\{\mathcal{Z}(\mu) \mid \mu \in \mathcal{P}\}$ is a basis for the topology of $\hat{\mathcal{E}}_{\infty}$;

(3) for $\mu, \rho \in \mathcal{P}$, the set $\mathcal{Z}(\mu) \backslash \mathcal{Z}(\rho)$ is a finite disjoint union of sets of the form $\mathcal{Z}\left(\mu^{\prime}\right)$, for $\mu^{\prime} \in \mathcal{P}$; and

(4) for each $\mu \in \mathcal{P}$, the set $\mathcal{Z}(\mu)$ is open and compact.

Proof. (1) If $\mu=\gamma \lambda$ and $\rho=\gamma^{\prime} \lambda^{\prime}$, where $\gamma, \lambda$ and $\gamma^{\prime}, \lambda^{\prime}$ are as in Definition 3.7, then $\mathcal{Z}(\mu) \cap \mathcal{Z}(\rho)=\emptyset$ except when either $\mu$ is an initial segment of $\rho$ (in which case $\mathcal{Z}(\rho) \subseteq \mathcal{Z}(\mu)$ ), or $\rho$ is an initial segment of $\mu$ (in which case $\mathcal{Z}(\mu) \subseteq \mathcal{Z}(\rho)$ ), or $\gamma=\gamma^{\prime}$ and $\lambda=\prod_{j=1}^{k(p)} \alpha(p, j)^{k_{j}}$, $\lambda^{\prime}=\prod_{j=1}^{k(p)} \alpha(p, j)^{l_{j}}$ for $k_{j}, l_{j} \in \mathbb{Z}^{+}, 1 \leq j \leq k(p)$, for a free prime $p$. In the latter case, we have

$$
\mathcal{Z}(\mu) \cap \mathcal{Z}(\rho)=\mathcal{Z}\left(\gamma \prod_{k=1}^{k(p)} \alpha(p, j)^{t_{j}}\right)
$$

where $t_{j}=\max \left\{k_{j}, l_{j}\right\}$, for $j=1, \ldots, k(p)$.

(2) This is [18, Proposition 2.5].

(3) Suppose that $\mathcal{Z}(\mu) \backslash \mathcal{Z}(\rho)$ is non-empty for a given pair $\mu, \rho \in \mathcal{P}$. Since

$$
\mathcal{Z}(\mu) \backslash \mathcal{Z}(\rho)=\mathcal{Z}(\mu) \backslash(\mathcal{Z}(\mu) \cap \mathcal{Z}(\rho))
$$

we may assume, using (1), that $\mathcal{Z}(\rho) \subset \mathcal{Z}(\mu)$, and hence that $\mu$ is an initial segment of $\rho$.

Thus, $\mathcal{Z}(\mu) \backslash \mathcal{Z}(\rho)$ is the set of infinite paths which have $\mu$ as initial segment but do not have $\rho$ as initial segment. Now, using that $E$ is row-finite, we see that it suffices to consider the situation where there is a finite path $\gamma$ ending at $v^{p}$ for some $p \in I_{\text {free}}$, and there are exponents $l_{j} \leq k_{j}$ for all $j$, with strict inequality for at least one of the indices $j \in\{1, \ldots, k(p)\}$, such that

$$
\mu=\gamma \prod_{j=1}^{k(p)} \alpha(p, j)^{l_{j}} \quad \text { and } \quad \rho=\gamma \prod_{j=1}^{k(p)} \alpha(p, j)^{k_{j}}
$$

We have

$$
\mathcal{Z}(\mu) \backslash \mathcal{Z}(\rho)=\bigsqcup_{\left\{j: l_{j}<k_{j}\right\}} \bigsqcup_{l_{j} \leq t_{j}<k_{j}} \bigsqcup_{s=1}^{g(p, j)} \mathcal{Z}\left(\gamma \alpha(p, j)^{t_{j}} \beta(p, j, s)\right)
$$


which gives the desired decomposition.

(4) This is a standard argument, which we review for the sake of completeness. The topology of $\hat{\mathcal{E}}_{0}$ is the topology induced by the embedding into the compact set $\{0,1\}^{\mathcal{E}}$. The sets of the form $\mathcal{U}(X, \emptyset)$, for $X \neq \emptyset$, are compact in $\hat{\mathcal{E}}_{0}$ because they are closed subsets of the basic compact and open subsets

$$
\left\{f \in\{0,1\}^{\mathcal{E}} \mid f(x)=1 \quad \forall x \in X\right\}
$$

of $\{0,1\}^{\mathcal{E}}$. (Note that the assumption $X \neq \emptyset$ is crucial here). Since the space $\hat{\mathcal{E}}_{\infty}$ is closed in $\hat{\mathcal{E}}_{0}$ (Theorem 3.6), we conclude that $\mathcal{U}^{\infty}(X, \emptyset)=\mathcal{U}(X, \emptyset) \cap \hat{\mathcal{E}}_{\infty}$ is compact in $\hat{\mathcal{E}}_{\infty}$.

For the next corollary, we need a straightforward lemma, probably well-known.

Lemma 3.9. Let $X$ be a topological space with a basis $\mathcal{B}$ of the topology satisfying the following properties:

(1) Each $B \in \mathcal{B}$ is a compact open subset of $X$.

(2) $\mathcal{B}$ is closed under finite intersections

(3) For each $B_{1}, B_{2} \in \mathcal{B}$, we have that $B_{1} \backslash B_{2}$ is a finite disjoint union of sets from $\mathcal{B}$.

Then every compact open subset of $X$ is a finite disjoint union of members of $\mathcal{B}$.

Proof. Let $U$ be a compact open subset of $X$. By compactness we can write $U=\bigcup_{i \in I} B_{i}$, where $I$ is finite and $B_{i} \in \mathcal{B}$. Now we can refine this decomposition to a disjoint decomposition into compact and open subsets of the form

$$
\left(B_{i_{1}} \cap B_{i_{2}} \cap \cdots \cap B_{i_{r}}\right) \backslash\left[\bigcup_{j \notin\left\{i_{1}, \ldots, i_{r}\right\}}\left(B_{i_{1}} \cap B_{i_{2}} \cap \cdots \cap B_{i_{r}} \cap B_{j}\right)\right],
$$

where $i_{1}, \ldots, i_{r}$ are different elements of $I$, and $1 \leq r \leq|I|$. (In case $r=|I|$ this term should be interpreted as $\bigcap_{i \in I} B_{i}$.) Now use the hypothesis to express each of these sets as a finite disjoint union of members of $\mathcal{B}$.

Corollary 3.10. The space $\hat{\mathcal{E}}_{\infty}$ of ultrafilters admits a basis of compact open subsets, namely the family $\{\mathcal{Z}(\mu)\}_{\mu \in \mathcal{P}}$. Moreover, every compact open subset of $\hat{\mathcal{E}}_{\infty}$ is a finite disjoint union of sets of the form $\mathcal{Z}(\mu)$, for $\mu \in \mathcal{P}$.

Proof. By Proposition 3.8, the family $\{\mathcal{Z}(\mu)\}_{\mu \in \mathcal{P}} \cup\{\emptyset\}$ satisfies the hypothesis of Lemma 3.9. Therefore the result follows from Lemma 3.9.

\section{The $K$-algebra $\mathcal{S}_{K}(E, C)$ induced by the inverse Semigroup $S(E, C)$}

Following the notation of section 2, given an adaptable graph $(E, C)$, we denote its associated inverse semigroup by $S(E, C)$, and it space of tight filters by $\widehat{\mathcal{E}}_{\text {tight }}$. By Corollary 3.5 and Theorem 3.6, the set of tight filters corresponds to the set of infinite paths. The main result of this section is Theorem 4.14, where we prove that the Steinberg algebra $A_{K}\left(\mathcal{G}_{\text {tight }}(E, C)\right)$ of the groupoid $\mathcal{G}_{\text {tight }}(E, C)$ of germs of the canonical action of $S(E, C)$ on $\widehat{\mathcal{E}}_{\text {tight }}$ is canonically isomorphic to the $*$-algebra $\mathcal{S}_{K}(E, C)$ defined by the family of generators $E^{0} \cup E^{1} \cup\left\{\left(t_{i}^{v}\right)^{ \pm}\right\}$ and the relations given in 2.2. In order to ease understanding this section we have divided it in three parts. Firstly we recall the construction of the groupoid $\mathcal{G}_{\text {tight }}(E, C)$. Then, we study the behaviour of $\mathcal{S}_{K}(E, C)$, and we finish the section proving Theorem 4.14. 
4.1. Steinberg algebras and inverse semigroup representations. In this subsection we explain the construction of the groupoid of germs of a partial action of an inverse semigroup $S$, and review the fact that the Steinberg algebra is the universal *-algebra for tight representations of $S$.

Let $S$ be an inverse semigroup with 0 , and denote by $\mathcal{E}$ its semilattice of idempotents. First recall that, if $F \subseteq \mathcal{E}$ is any subset, then a finite subset $\Sigma \subseteq F$ is a finite cover of $F$ when for any $0 \neq f \in F$ there exists $e \in \Sigma$ such that $f e \neq 0$.

Definition 4.1. (cf. [17], [35, Section 5]) Let $S$ be an inverse semigroup, and $A$ be a *algebra over a field with involution $K$. Then, we say that $\pi: S \rightarrow A$ is a representation if $\pi(s t)=\pi(s) \pi(t)$ and $\pi\left(s^{*}\right)=\pi(s)^{*}$ for all $s, t \in S$. A representation $\pi$ is said to be a tight representation if for every idempotent $e \in \mathcal{E}$ and every finite cover $Z$ of $\mathcal{F}_{e}:=\{f \in \mathcal{E} \mid f \leq e\}$, we have

$$
\pi(e)=\bigvee_{z \in Z} \pi(z)
$$

in the (generalized) Boolean algebra of idempotents of the commutative $*$-subalgebra $A_{\mathcal{E}}$ of $A$ generated by $\pi(\mathcal{E})$.

Given a semilattice $\mathcal{E}$ with 0 , the space $\widehat{\mathcal{E}}$ is the space of semicharacters on $\mathcal{E}$, that is the space of non-zero semilattice homomorphisms $\varphi: \mathcal{E} \rightarrow\{0,1\}$, endowed with the relative topology from $\{0,1\}^{\mathcal{E}}$. The space of characters $\widehat{\mathcal{E}}_{0}$ is the space of those semicharacters $\varphi$ such that $\varphi\left(0_{S}\right)=0$ [17, Definition 12.4], and may be identified with the space of filters over $\mathcal{E}$ (see Definition 3.1) via the map that associates to each filter $F$ its characteristic function $1_{F}: \mathcal{E} \rightarrow\{0,1\}$. A character $\varphi$ is said to be tight if $\varphi(e)=\bigvee_{f \in Z} \varphi(f)$ for every idempotent $e \in \mathcal{E}$ and every finite cover $Z$ of $\mathcal{F}_{e}$. By [17, Proposition 11.8], this is equivalent to the original definition of tight character in [17, Definition 12.8]. The space of tight characters will be identified with the space of tight filters, and will be denoted by $\widehat{\mathcal{E}}_{\text {tight }}$ in accordance with the notation employed in Section 3.

We recall below the construction of the groupoid of germs for completeness (see [17] for further details). If $\alpha$ is an action of an inverse semigroup $S$ on a locally compact Hausdorff space $X$, then the groupoid of germs $S \times{ }_{\alpha} X$ is defined as follows: define a relation $\sim$ on $\left\{(s, x) \in S \times X: x \in \operatorname{dom}\left(\alpha_{s}\right)\right\}$ by $(s, x) \sim\left(s^{\prime}, y\right)$ if $x=y$ and there is an idempotent $p \in E(S)$ such that $x \in \operatorname{dom}\left(\alpha_{p}\right)$ and $s p=s^{\prime} p$. This is an equivalence relation, and the collection $S \times{ }_{\alpha} X$ of equivalence classes for this relation is a locally compact étale groupoid with unit space $X$ and structure maps $r([s, x])=\alpha_{s}(x), s([s, x])=x,\left[s, \alpha_{t}(x)\right][t, x]=[s t, x]$, and $[s, x]^{-1}=\left[s^{*}, \alpha_{s}(x)\right]$. When $X$ is the space $\widehat{\mathcal{E}}_{\text {tight }}$ of tight filters on the semilattice $\mathcal{E}$ of idempotents of $S$, the topology of the groupoid of germs $\mathcal{G}_{\text {tight }}(S):=S \times_{\alpha} \widehat{\mathcal{E}}_{\text {tight }}$ is generated by the sets

$$
\Theta(s, U):=\{[s, x] \in \mathcal{G} \mid x \in U\},
$$

where $U$ is an open subset of $\widehat{\mathcal{E}}_{\text {tight }}$ such that $U \subseteq D_{s^{*} s}:=\left\{\varphi \in \widehat{\mathcal{E}}_{\text {tight }} \mid \varphi\left(s^{*} s\right)=1\right\}$. Endowed with this topology, $\mathcal{G}_{\text {tight }}(S)$ is an ample groupoid. Recall that the canonical action $\alpha$ of $S$ on $\widehat{\mathcal{E}}_{\text {tight }}$ is given as follows. For $s \in S$, the map $\alpha_{s}: D_{s^{*} s} \rightarrow D_{s s^{*}}$ is the map defined by $\alpha_{s}(\varphi)(e)=\varphi\left(s^{*} e s\right)$ for all $\varphi \in D_{s^{*} s}$.

Now, given an inverse semigroup $S$ and a field with involution $K$, we may consider the Steinberg $K$-algebra $A_{K}\left(\mathcal{G}_{\text {tight }}(S)\right)$ associated to the ample groupoid $\mathcal{G}_{\text {tight }}(S)$ (Definition 1.5). 
If $\mathcal{G}_{\text {tight }}(S)$ is Hausdorff, then the algebra $A_{K}\left(\mathcal{G}_{\text {tight }}(S)\right)$ is just the algebra of $K$-valued locally constant functions with compact support, endowed with the convolution product and the involution $f^{*}(\alpha)=f\left(\alpha^{-1}\right)^{*}$.

We say that a $*$-algebra $A$, together with a tight representation $\iota: S \rightarrow A$, is universal for tight representations if given any $*$-algebra $B$ and any tight representation $\phi: S \rightarrow B$, there is a unique $*$-homomorphism $\tilde{\phi}: A \rightarrow B$ such that $\tilde{\phi} \circ \iota=\phi$. By the usual argument, such a universal tight $*$-algebra is unique up to $*$-isomorphism.

We will need the following result, due to Steinberg [35].

Theorem 4.2. [35, Corollary 5.3] Let $S$ be a Hausdorff inverse semigroup with zero and let $K$ be a field with involution. Then $A_{K}\left(\mathcal{G}_{\text {tight }}(S)\right)$ is the universal *-algebra for tight representations of $S$.

We refer the reader to [35] for the definition of a Hausdorff inverse semigroup. For us, it is enough to know that any $E^{*}$-unitary inverse semigroup is Hausdorff (see [35, page 1037]).

4.2. The *-algebra $\mathcal{S}_{K}(E, C)$ and expansions. We define the $K$-algebra $\mathcal{S}_{K}(E, C)$ as the *-algebra over $K$ with generators $E^{0} \cup E^{1} \cup\left\{\left(t_{i}^{v}\right)^{ \pm}: i \in \mathbb{N}, v \in E^{0}\right\}$ and defining relations given in $\mathbf{2 . 2}$ (including this time all the relations); notice that $\mathcal{S}_{K}(E, C)$ is the $K$-span of the elements of the inverse semigroup $S(E, C)$. We will show that the natural representation of $S(E, C)$ inside $\mathcal{S}_{K}(E, C)$ is its universal tight representation (Theorem 4.12, Theorem 4.13).

Let us start fixing the notation $\iota: S(E, C) \rightarrow \mathcal{S}_{K}(E, C)$ for the natural representation of $S(E ; C)$ into $\mathcal{S}_{K}(E, C)$, and recalling that all relevant notions have been defined in Section 4.1.

Remark 4.3. There are a couple of equalities that play a key role in checking tightness of $\iota$. We recall them below for convenience.

(a) for each $p \in I_{\text {reg }}$ and each $v \in E_{p}^{0}$,

$$
v=\sum_{e \in s^{-1}(v)} e e^{*}
$$

(b) for each $p \in I_{\text {free }}$ and each $1 \leq i \leq k(p)$,

$$
v^{p}=\alpha(p, i) \alpha(p, i)^{*}+\sum_{s=1}^{g(p, i)} \beta(p, i, s) \beta(p, i, s)^{*}
$$

Let us start by establishing the following result about suprema in $\mathcal{S}_{K}(E, C)$ :

Lemma 4.4. For $p \in I_{\text {free }}$, let $\mathbf{m}_{1}, \mathbf{m}_{2} \in \mathcal{E}$ monomials associated to $p$. Then $\iota\left(\mathbf{m}_{1} \vee \mathbf{m}_{2}\right)=$ $\iota\left(\mathbf{m}_{1}\right) \vee \iota\left(\mathbf{m}_{2}\right)$.

Proof. We can describe $\mathbf{m}_{1}$ and $\mathbf{m}_{2}$ as follows:

$$
\mathbf{m}_{1}=\prod_{i=1}^{k(p)} \alpha(p, i)^{k_{i}}\left(\alpha(p, i)^{*}\right)^{k_{i}} \text { and } \mathbf{m}_{2}=\prod_{i=1}^{k(p)} \alpha(p, i)^{l_{i}}\left(\alpha(p, i)^{*}\right)^{l_{i}}
$$

Let $s_{i}:=\max \left\{k_{i}, l_{i}\right\}$ and $r_{i}:=\min \left\{k_{i}, l_{i}\right\}$ for $1 \leq i \leq k(p)$. Then

$$
\mathbf{m}_{1} \cdot \mathbf{m}_{2}=\prod_{i=1}^{k(p)} \alpha(p, i)^{s_{i}}\left(\alpha(p, i)^{*}\right)^{s_{i}} \text { and } \mathbf{m}_{1} \vee \mathbf{m}_{2}=\prod_{i=1}^{k(p)} \alpha(p, i)^{r_{i}}\left(\alpha(p, i)^{*}\right)^{r_{i}}
$$


By using the expansion rule explained in Remark 4.3 (b) as much as needed, we have:

$$
\begin{aligned}
\iota\left(\mathbf{m}_{1} \vee \mathbf{m}_{2}\right)=\mathbf{m}_{1} \mathbf{m}_{2} & +\sum_{k_{i}<l_{i}} \sum_{r=1}^{g(p, i)} \sum_{t=1}^{l_{i}-k_{i}-1} \alpha(p, i)^{k_{i}+t} \beta(p, i, r) \beta(p, i, r)^{*}\left(\alpha(p, i)^{*}\right)^{k_{i}+t} \\
& +\sum_{l_{j}<k_{j}} \sum_{r=1}^{g(p, j)} \sum_{t=1}^{k_{j}-l_{j}-1} \alpha(p, j)^{l_{j}+t} \beta(p, j, r) \beta(p, j, r)^{*}\left(\alpha(p, j)^{*}\right)^{l_{j}+t},
\end{aligned}
$$

where the latter computation is performed in the algebra $\mathcal{S}_{K}(E, C)$.

Now, we have:

(1) $\left[\iota\left(\mathbf{m}_{1}\right) \vee \iota\left(\mathbf{m}_{2}\right)\right]\left(\mathbf{m}_{1} \mathbf{m}_{2}\right)=\mathbf{m}_{1} \mathbf{m}_{2}$

(2) For each summand where $k_{i}<l_{i}$,

$$
\begin{aligned}
{\left[\iota\left(\mathbf{m}_{1}\right)\right.} & \left.\vee \iota\left(\mathbf{m}_{2}\right)\right]\left(\sum_{r=1}^{g(p, i)} \sum_{t=1}^{l_{i}-k_{i}-1} \alpha(p, i)^{k_{i}+t} \beta(p, i, r) \beta(p, i, r)^{*}\left(\alpha(p, i)^{*}\right)^{k_{i}+t}\right) \\
& =\left(\mathbf{m}_{1}+\mathbf{m}_{2}-\mathbf{m}_{1} \mathbf{m}_{2}\right)\left(\sum_{r=1}^{g(p, i)} \sum_{t=1}^{l_{i}-k_{i}-1} \alpha(p, i)^{k_{i}+t} \beta(p, i, r) \beta(p, i, r)^{*}\left(\alpha(p, i)^{*}\right)^{k_{i}+t}\right) \\
& =(a)
\end{aligned}
$$

Fixing $r$ and $t$, we have

$$
\begin{aligned}
\mathbf{m}_{1}\left(\alpha(p, i)^{k_{i}+t} \beta\right. & \left.(p, i, r) \beta(p, i, r)^{*}\left(\alpha(p, i)^{*}\right)^{k_{i}+t}\right) \\
& =\prod_{j=1}^{k(p)} \alpha(p, j)^{k_{j}}\left(\alpha(p, j)^{*}\right)^{k_{j}}\left(\alpha(p, i)^{k_{i}+t} \beta(p, i, r) \beta(p, i, r)^{*}\left(\alpha(p, i)^{*}\right)^{k_{i}+t}\right) \\
& =\alpha(p, i)^{k_{i}+t} \beta(p, i, r) \beta(p, i, r)^{*}\left(\alpha(p, i)^{*}\right)^{k_{i}+t} .
\end{aligned}
$$

Indeed, for $j \neq i$, we have an absorption of the $j$-th part and, for $i=j$, one has $\alpha(p, i)^{k_{i}}\left(\alpha(p, i)^{*}\right)^{k_{i}} \alpha(p, i)^{k_{i}+t}=\alpha(p, i)^{k_{i}+t}$.

The same product for $\mathbf{m}_{2}$ equals 0 since $k_{i}+t<l_{i}$. And, of course, the same happens for $\mathbf{m}_{1} \mathbf{m}_{2}$. Therefore,

$$
(a)=\sum_{r=1}^{g(p, i)} \sum_{t=1}^{l_{i}-k_{i}-1} \alpha(p, i)^{k_{i}+t} \beta(p, i, r) \beta(p, i, r)^{*}\left(\alpha(p, i)^{*}\right)^{k_{i}+t} .
$$

(3) For the summands where $k_{j}<l_{j}$, an analogous argument works.

Therefore, $\left(\iota\left(\mathbf{m}_{1}\right) \vee \iota\left(\mathbf{m}_{2}\right)\right) \cdot\left(\iota\left(\mathbf{m}_{1} \vee \mathbf{m}_{2}\right)\right)=\iota\left(\mathbf{m}_{1} \vee \mathbf{m}_{2}\right)$, whence $\iota\left(\mathbf{m}_{1} \vee \mathbf{m}_{2}\right) \leq \iota\left(\mathbf{m}_{1}\right) \vee \iota\left(\mathbf{m}_{2}\right)$. Since the reverse inequality is obvious, we are done.

Let us now introduce some properties for finite covers $\Sigma$ of $\mathcal{F}_{e}$.

Definition 4.5. Given $\Sigma \subset \mathcal{F}_{e}$ a finite cover, we will say that $\Sigma$ is irredundant if for all $f \neq g \in \Sigma$, we have that $f$ is orthogonal to $g$, i.e. $f g=0$. 
Proposition 4.6. Let $e \in \mathcal{E}$ and $\Sigma \subset \mathcal{F}_{e}$ a finite cover of $\mathcal{F}_{e}$. Then there exists an irredundant finite cover $\hat{\Sigma}$ such that

$$
\bigvee_{f \in \Sigma} \iota(f)=\bigvee_{g \in \hat{\Sigma}} \iota(g)
$$

Proof. We proceed by induction on the cardinality of $\Sigma$. If $|\Sigma|=1$, then the result is obvious. Assume that $|\Sigma|>1$ and the result holds for finite covers of cardinality less than $|\Sigma|$. Suppose that there are $f, g \in \Sigma$ such that $f g \neq 0$. By induction, it suffices to show that there is a finite cover $\Sigma^{\prime}$ of $\mathcal{F}_{e}$, with $\left|\Sigma^{\prime}\right|<|\Sigma|$, such that $\bigvee_{h \in \Sigma} \iota(h)=\bigvee_{h \in \Sigma^{\prime}} \iota(h)$. By Lemma 2.17, we have that either $f$ and $g$ are comparable, or there is a free prime $p$ such that $f=\gamma \mathbf{m}(p) \gamma^{*}$ and $g=\gamma \mathbf{m}^{\prime}(p) \gamma^{*}$ for some finite path $\gamma$ and monomials $\mathbf{m}(p)$ and $\mathbf{m}^{\prime}(p)$ at $p$. If $f$ and $g$ are comparable, for instance $f \leq g$, then we consider the finite cover $\Sigma^{\prime}=\Sigma \backslash\{f\}$. In case $f$ and $g$ are not comparable, then set

$$
\Sigma^{\prime}:=(\Sigma \backslash\{f, g\}) \cup\left\{f^{\prime}\right\}
$$

where $f^{\prime}=\gamma\left(\mathbf{m}(p) \vee \mathbf{m}^{\prime}(p)\right) \gamma^{*}$. By Lemma 4.4, we have that $\iota\left(f^{\prime}\right)=\iota(f) \vee \iota(g)$, and thus $\vee_{h \in \Sigma^{\prime}} \iota(h)=\vee_{h \in \Sigma^{\prime}}(h)$, concluding the proof.

We now introduce a crucial concept for our construction.

Definition 4.7. If $\sum e_{i}$ is a finite orthogonal sum of idempotents $e_{i} \in \mathcal{E}$ in $\mathcal{S}_{K}(E, C)$, then a simple expansion of $\sum e_{i}$ consists of another orthogonal sum of the form $\sum_{z \in Z} e_{z}^{\prime}+\sum_{i \neq i_{0}} e_{i}$, for some $i_{0}$, obtained by applying the rules described in Remark 4.3 to $e_{i_{0}}$; concretely, the idempotents $e_{z}^{\prime} \in \mathcal{E}, z \in Z$, are of one of the following forms:

(1) If $e_{i_{0}}=\gamma \prod_{j=1}^{k(p)} \alpha(p, j)^{k_{j}}\left(\alpha(p, j)^{*}\right)^{k_{j}} \gamma^{*}$ for a free prime $p$, then

$$
\begin{aligned}
\sum_{z \in Z} e_{z}^{\prime}=\gamma\left[\alpha\left(p, j_{0}\right)^{k_{j_{0}}+1}(\right. & \left.\left.\alpha\left(p, j_{0}\right)^{*}\right)^{k_{j_{0}}+1} \prod_{j \neq j_{0}} \alpha(p, j)^{k_{j}}\left(\alpha(p, j)^{*}\right)^{k_{j}}\right] \gamma^{*} \\
& +\sum_{s=1}^{g\left(p, j_{0}\right)}\left[\gamma \alpha\left(p, j_{0}\right)^{k_{j_{0}}} \beta\left(p, j_{0}, s\right) \beta\left(p, j_{0}, s\right)^{*}\left(\alpha\left(p, j_{0}\right)^{*}\right)^{k_{j_{0}}} \gamma^{*}\right]
\end{aligned}
$$

for some $1 \leq j_{0} \leq k(p)$.

(2) If $e_{i_{0}}=\gamma \lambda \lambda^{*} \gamma^{*}$ for some path of finite length $\lambda$ in the graph $E_{p}$, where $p$ is a regular prime, then $Z=s^{-1}(r(\lambda))$, and

$$
e_{z}^{\prime}=\gamma \lambda z z^{*} \lambda^{*} \gamma^{*} \quad(z \in Z)
$$

Definition 4.8. An expansion of $e \in \mathcal{E}$ in $\mathcal{S}_{K}(E, C)$ is any expression obtained by applying a finite number of simple expansions to the original expression of $e$. We define the expanded set of the given expression as the set of orthogonal idempotents in $\mathcal{E}$ that appear in the expansion. 
Example 4.9. Let $e=\gamma \mathbf{m}(p) \gamma^{*}$ be a projection in $\mathcal{E}$, with $p$ a free prime in $I, \gamma$ a finite path to $v^{p}$ and $\mathbf{m}(p)=\alpha(p, i) \alpha(p, i)^{*}$ for some $i \in\{1, \ldots, k(p)\}$. Then

$$
\begin{aligned}
e & =\gamma \alpha(p, i) \alpha(p, i)^{*} \gamma^{*}=\gamma \alpha(p, i) v^{p} \alpha(p, i)^{*} \gamma^{*} \\
& =\gamma \alpha(p, i) \alpha(p, i) \alpha(p, i)^{*} \alpha(p, i)^{*} \gamma^{*}+\gamma \alpha(p, i)\left[\sum_{s=1}^{g(p, i)} \beta(p, i, s) \beta(p, i, s)^{*}\right] \alpha(p, i)^{*} \gamma^{*} \\
& =\gamma \alpha(p, i) \alpha(p, i) \alpha(p, i)^{*} \alpha(p, i)^{*} \gamma^{*}+\sum_{s=1}^{g(p, i)}\left[\gamma \alpha(p, i) \beta(p, i, s) \beta(p, i, s)^{*} \alpha(p, i)^{*} \gamma^{*}\right] .
\end{aligned}
$$

The latter equation is what we call an expansion of $e$. The expanded set of the above expression contains the projections $\gamma \alpha(p, i) \alpha(p, i) \alpha(p, i)^{*} \alpha(p, i)^{*} \gamma^{*}$ and also each of the projections $\gamma \alpha(p, i) \beta(p, i, s) \beta(p, i, s)^{*} \alpha(p, i)^{*} \gamma^{*}$.

A consequence of the above definition is the following.

Lemma 4.10. If $\Sigma$ is an expanded set of $e \in \mathcal{E}$, then it is an irredundant finite cover of $\mathcal{F}_{e}$.

Proof. If $\Sigma$ is an expanded set of $e \in \mathcal{E}$, then $\Sigma \subset \mathcal{F}_{e}$, and its elements are pairwise orthogonal. We will show that for any idempotent $f \leq e$ and any expanded set $\Sigma$ of $e$, either $f \leq g$ for some $g \in \Sigma$ or $g \leq f$ for some $g \in \Sigma$. We proceed by induction on the number of simple expansions (Definition 4.7) used to get $\Sigma$ from $e$. If the number of simple expansions is 0 , then $\Sigma=\{e\}$ and $f \leq e$, as desired. Assume the result holds for expansions obtained by using $n-1$ simple expansions of $e$, for $n \geq 1$, and let $\Sigma$ be an expansion of $e$ obtained by using $n$ simple expansions. Then there exists an expansion $\Sigma^{\prime}$ obtained from $e$ by using $n-1$ simple expansions and an element $g^{\prime}$ of $\Sigma^{\prime}$ such that

$$
\Sigma=\left(\Sigma^{\prime} \backslash\left\{g^{\prime}\right\}\right) \cup\left\{g_{1}, g_{2}, \ldots, g_{N}\right\} .
$$

Indeed, write $g^{\prime}=\gamma \mathbf{m}(p) \gamma^{*}$ in its standard form. If $p$ is a free prime, then write $g^{\prime}=$ $\gamma \prod_{j=1}^{k} \alpha(p, j)^{k_{j}}\left(\alpha(p, j)^{*}\right)^{k_{j}} \gamma^{*}$ for $k_{j} \in \mathbb{Z}^{+}$, and, for some $1 \leq i \leq k(p)$, we have

$$
g_{1}=\gamma \alpha(p, i)^{k_{i}+1}\left(\alpha(p, i)^{*}\right)^{k_{i}+1} \prod_{j \neq i} \alpha(p, j)^{k_{j}}\left(\alpha(p, j)^{*}\right)^{k_{j}} \gamma^{*}
$$

and

$$
g_{s+1}=\gamma \alpha(p, i)^{k_{i}} \beta(p, i, s) \beta(p, i, s)^{*}\left(\alpha(p, i)^{*}\right)^{k_{i}} \gamma^{*},
$$

for $1 \leq s \leq g(p, i)$, where $N=g(p, i)+1$. If $p$ is a regular prime, one has

$$
g^{\prime}=\gamma \lambda \lambda^{*} \gamma^{*}
$$

where $\lambda$ is a path of finite length in the graph $E_{p}$, and

$$
g_{i}=\gamma \lambda e_{i} e_{i}^{*} \lambda^{*} \gamma^{*}
$$

with $s^{-1}(r(\lambda))=\left\{e_{1}, \ldots, e_{N}\right\}$.

By the induction hypothesis, there is some $g^{\prime \prime} \in \Sigma^{\prime}$ such that either $f \leq g^{\prime \prime}$ or $g^{\prime \prime} \leq f$. Now, if $g^{\prime} \neq g^{\prime \prime}$, then we take $g=g^{\prime \prime}$ and either $f \leq g$ or $g \leq f$. So assume that $g^{\prime}=g^{\prime \prime}$. If $g^{\prime} \leq f$ then clearly $g_{i} \leq f$ for $i=1,2, \ldots, N$, so we can take $g$ to be any of them. Finally assume that $f<g^{\prime}$. When $p$ is a free prime, this means that $f=\gamma \gamma^{\prime} \mathbf{n}\left(p^{\prime}\right)\left(\gamma^{\prime}\right)^{*} \gamma^{*}$, where $\gamma^{\prime}$ is a finite path from $v^{p}$ to $w \in E_{p^{\prime}}^{0}$. If $\gamma^{\prime}$ is a trivial finite path, then $\mathbf{n}\left(p^{\prime}\right)=\prod \alpha(p, j)^{l_{j}}\left(\alpha(p, j)^{*}\right)^{l_{j}}$ and 
necessarily we have that $l_{j} \geq k_{j}$ for all $j$. Now if $l_{i} \geq k_{i}+1$, then we get $f \leq g_{1}$, so we can take $g=g_{1}$. If $l_{i}=k_{i}$, then $g_{s+1} \leq f$ for $s=1, \ldots, g(p, i)$, so we can take $g$ to be any of them. Assume now that $\gamma^{\prime}$ is non-trivial and write $\gamma^{\prime}=\alpha\left(p, i^{\prime}\right)^{m} \beta\left(p, i^{\prime}, s\right) \cdots$, for some $i^{\prime} \in\{1, \ldots, k(p)\}$ and some $s \in\left\{1, \ldots, g\left(p, i^{\prime}\right)\right\}$. Since $f \leq g^{\prime}$, we must have $m \geq k_{i^{\prime}}$. Now if $i \neq i^{\prime}$, then we will have $f \leq g_{1}$, and we take $g=g_{1}$. If $i=i^{\prime}$ and $m=k_{i}$, then $f \leq g_{s+1}$ and we take $g=g_{s+1}$. Finally, if $i=i^{\prime}$ and $m>k_{i}$, then $f \leq g_{1}$ and we take $g=g_{1}$.

If $p$ is a regular prime, then since $f<g^{\prime}$, there exists $e_{i} \in s^{-1}(r(\lambda))$ such that $f \leq$ $\gamma \lambda e_{i} e_{i}^{*} \lambda^{*} \gamma^{*}=g_{i}$.

The next result shows that the converse of Lemma 4.10 also holds.

Proposition 4.11. Let $e \in \mathcal{E}$, and let $\Sigma \subset \mathcal{F}_{e}$ be an irredundant finite cover of $\mathcal{F}_{e}$. Then, $\Sigma$ is an expanded set of $e$.

Proof. We will proceed by induction on the number of elements of $\Sigma$. The result is clear if $|\Sigma|=1$. Assume that $|\Sigma|>1$ and the result holds for finite irredundant covers of idempotents in $\mathcal{E}$ of cardinality less than $|\Sigma|$.

Suppose first that $e=\gamma \prod_{i=1}^{k(p)} \alpha(p, i)^{k_{i}}\left(\alpha(p, i)^{*}\right)^{k_{i}} \gamma^{*}$, where $p$ is a free prime. We first show that $\Sigma$ must contain some element $e^{\prime}$ of the form $e^{\prime}=\gamma \prod_{i=1}^{k(p)} \alpha(p, i)^{l_{i}}\left(\alpha(p, i)^{*}\right)^{l_{i}} \gamma^{*}$, where $l_{i}>k_{i}$ for at least one index $i$. Assume not. Then each $f \in \Sigma$ is of the form $\gamma \gamma^{\prime} \mathbf{m}\left(\gamma^{\prime}\right)^{*} \gamma^{*}$, where $\gamma^{\prime}=\alpha(p, i(f))^{m(f)} \beta(p, i(f), s(f)) \cdots$ for some non-negative integers $m(f)$ and positive integers $s(f)$. Let $\Pi=\{i(f) \mid f \in \Sigma\} \subseteq\{1, \ldots, k(p)\}$ be the set of indices appearing in the starting terms of the elements of $\Sigma$. For each $i \in \Pi$, take an integer $M_{i}$ such that $M_{i}>m(f)$ for every $f \in \Sigma$ such that $i(f)=i$. Note that $M_{i}>k_{i}$ for $i \in \Pi$. If $i \notin \Pi$, set $M_{i}=k_{i}$. Now consider $f \in \mathcal{E}$ defined by

$$
f=\gamma \prod_{i=1}^{k(p)} \alpha(p, i)^{M_{i}}\left(\alpha(p, i)^{*}\right)^{M_{i}} \gamma^{*}
$$

Then $0 \neq f \in \mathcal{F}_{e}$ and $f g=0$ for each $g \in \Sigma$, which is a contradiction. Therefore there is an element $e^{\prime}$ in $\Sigma$ of the form described above, and it is necessarily unique, because $\Sigma$ is irredundant, and the product of idempotents of this form is always nonzero. Note that $e^{\prime} \neq e$ because $|\Sigma|>1$ and thus there is at least one index $i$ such that $l_{i}>k_{i}$.

Now we consider the following expansion of $e$ in $\mathcal{S}_{K}(E, C)$ leading to $e^{\prime}$ :

$$
e=e^{\prime}+\sum_{k_{i}<l_{i}} \sum_{t=0}^{l_{i}-k_{i}-1} \sum_{s=1}^{g(p, i)} \alpha(p, i)^{k_{i}+t} \beta(p, i, s) \beta(p, i, s)^{*}\left(\alpha(p, i)^{*}\right)^{k_{i}+t} .
$$

Let

$$
\Omega=\left\{\alpha(p, i)^{k_{i}+t} \beta(p, i, s) \beta(p, i, s)^{*}\left(\alpha(p, i)^{*}\right)^{k_{i}+t} \mid k_{i}<l_{i}, 0 \leq t \leq l_{i}-k_{i}-1,1 \leq s \leq g(p, i)\right\}
$$

and observe that $\Omega \cup\left\{e^{\prime}\right\}$ is an expanded set of $e$. The proof of Lemma 4.10 shows that for any $g \in \Sigma \backslash\left\{e^{\prime}\right\}$ there exists $f \in \Omega$ such that either $g \leq f$ of $f \leq g$. But now if $f \leq g$ for $f \in \Omega$ and $g \in \Sigma \backslash\left\{e^{\prime}\right\}$ then necessarily $f=g$. Thus, in any case, for each $g \in \Sigma \backslash\left\{e^{\prime}\right\}$ there is $f \in \Omega$ such that $g \leq f$. Since the elements of $\Omega$ are pairwise orthogonal, there is exactly one $f \in \Omega$ such that $g \leq f$.

For $f \in \Omega$, let $\Sigma_{f}=\left\{g \in \Sigma \backslash\left\{e^{\prime}\right\} \mid g \leq f\right\}$. By the above argument, $\left\{\Sigma_{f} \mid f \in \Omega\right\}$ is a partition of $\Sigma \backslash\left\{e^{\prime}\right\}$, Moreover, it follows easily that $\Sigma_{f}$ is an irredundant finite cover of $f$ for 
each $f \in \Omega$. By the induction hypothesis, $\Sigma_{f}$ is an expanded set of $f$, for $f \in \Omega$. Hence $\Sigma$ is an expanded set of $e$. This shows the result in the case where $p$ is a free prime.

Assume now that $p$ is a regular prime, and write $e=\gamma \lambda \lambda^{*} \gamma^{*}$, where $\gamma$ is a finite path and $\lambda$ is a path of finite length in the graph $E_{p}$. By a similar argument as before, there is $e^{\prime} \in \Sigma$ of the form $e^{\prime}=\gamma \lambda \lambda^{\prime}\left(\lambda^{\prime}\right)^{*} \lambda^{*} \gamma^{*}$, where $\lambda^{\prime}$ is a path of finite length in $E_{p}$. We assume that $\lambda^{\prime}$ has maximal length, say $r$, amongst all the paths in $E_{p}$ giving rise to an element of $\Sigma$. Write $\lambda^{\prime}=e_{1} e_{2} \cdots e_{r}$, where $e_{i} \in E_{p}^{1}$. We consider the following expansion of $e$ in $\mathcal{S}_{K}(E, C)$ :

$$
e=e^{\prime}+\sum_{i=0}^{r-1} \sum_{f \neq e_{i+1}, s(f)=s\left(e_{i+1}\right)} \gamma \lambda e_{1} \cdots e_{i} f f^{*} e_{i}^{*} \cdots e_{1}^{*} \lambda^{*} \gamma^{*}
$$

Let

$$
\Omega=\left\{\gamma \lambda e_{1} \cdots e_{i} f f^{*} e_{i}^{*} \cdots e_{1}^{*} \lambda^{*} \gamma^{*} \mid i=0, \ldots, r-1, f \in E^{1}, f \neq e_{i+1}, s(f)=s\left(e_{i+1}\right)\right\} .
$$

Then $\Omega \cup\left\{e^{\prime}\right\}$ is an expanded set of $e$. Therefore, by the proof of Lemma 4.10, for any $g \in \Sigma \backslash\left\{e^{\prime}\right\}$ there exists $h \in \Omega$ such that either $g \leq h$ or $h \leq g$. If $h \leq g$, where $h=$ $\gamma \lambda e_{1} \cdots e_{i} f f^{*} e_{i}^{*} \cdots e_{1}^{*} \lambda^{*} \gamma^{*}$ and $h \neq g$, then $g=\gamma \lambda e_{1} \cdots e_{j} e_{j}^{*} \cdots e_{1}^{*} \lambda^{*} \gamma^{*}$ for some $j \leq i$, forcing $g e^{\prime} \neq 0$, and contradicting irredundancy of $\Sigma$. Thus, in any case, for each $g \in \Sigma \backslash\left\{e^{\prime}\right\}$ there is $h \in \Omega$ such that $g \leq h$. Now the proof ends exactly as in the case where $p$ is a free prime.

Now, we are ready to show that the map $\iota$ is tight.

Theorem 4.12. The map $\iota: S(E, C) \rightarrow \mathcal{S}_{K}(E, C)$ is tight.

Proof. Let $e \in \mathcal{E}$, and $\Sigma \subset \mathcal{F}_{e}$ be a finite cover. Then, by Proposition 4.6, there exists an irredundant finite cover $\hat{\Sigma}$ of $\mathcal{F}_{e}$ such that

$$
\bigvee_{f \in \Sigma} \iota(f)=\bigvee_{g \in \hat{\Sigma}} \iota(g)
$$

By Proposition 4.11, $\hat{\Sigma}$ is an expanded set of an expression of $e$ in $\mathcal{S}_{K}(E, C)$, whence

$$
\iota(e)=\sum_{g \in \hat{\Sigma}} \iota(g) \quad \text { in } \quad \mathcal{S}_{K}(E, C) .
$$

Because the elements in $\hat{\Sigma}$ are pairwise orthogonal,

$$
\sum_{g \in \hat{\Sigma}} \iota(g)=\bigvee_{g \in \hat{\Sigma}} \iota(g) .
$$

Hence

$$
\iota(e)=\sum_{g \in \hat{\Sigma}} \iota(g)=\bigvee_{g \in \hat{\Sigma}} \iota(g)=\bigvee_{f \in \Sigma} \iota(f) .
$$

4.3. Universality of the Representation. In this subsection, we will show that the tight representation $\iota$ of $S(E, C)$ described above is universal for tight representations.

Theorem 4.13. The map $\iota: S(E, C) \rightarrow \mathcal{S}_{K}(E, C)$ is universal for tight representations of $S(E, C)$ on *-algebras over $K$. 
Proof. Let $A$ be a $*$-algebra, $\phi: S(E, C) \rightarrow A$ be a tight representation and denote by $\bar{x}$ the images of the elements of $S(E, C)$ under $\phi$. Since $\phi$ is tight, Definition 4.1 and Lemma 4.10 show that

(1) for each $p \in I_{\text {reg }}$ and each $\in E_{p}^{0}$,

$$
\bar{v}=\sum_{e \in s^{-1}(v)} \bar{e} \cdot \bar{e}^{*}, \text { and }
$$

(2) for each $p \in I_{\text {free }}$ and each $1 \leq i \leq k(p)$,

$$
\overline{v^{p}}=\overline{\alpha(p, i)} \cdot \overline{\alpha(p, i)}^{*}+\sum_{s=1}^{g(p, i)} \overline{\beta(p, i, s)} \cdot \overline{\beta(p, i, s)}^{*} .
$$

All other relations in $\mathcal{S}_{K}(E, C)$ are preserved because $\phi$ is a representation. Thus there exists a unique $K$-algebra $*$-homomorphism $\Phi: \mathcal{S}_{K}(E, C) \rightarrow A$ such that $\Phi(\iota(x))=\phi(x)$ for each generator $x$ of $S(E, C)$.

Using Theorem 4.13 and the results of Section 4.1, we obtain an isomorphism between the $K$-algebra $\mathcal{S}_{K}(E, C)$ and the Steinberg algebra $A_{K}\left(\mathcal{G}_{\text {tight }}(S(E, C))\right)$.

Theorem 4.14. Let $(E, C)$ be an adaptable separated graph, let $S(E, C)$ be the inverse semigroup associated to $(E, C)$, let $K$ be a field with involution and let $\mathcal{S}_{K}(E, C)$ be the *-algebra over $K$ associated to $(E, C)$. Let $A_{K}\left(\mathcal{G}_{\text {tight }}(S(E, C))\right)$ be the Steinberg algebra of the tight groupoid $\mathcal{G}_{\text {tight }}(S(E, C))$. There is a *-isomorphism

$$
\mathcal{S}_{K}(E, C) \cong A_{K}\left(\mathcal{G}_{\text {tight }}(S(E, C))\right)
$$

sending $\iota(s) \in \mathcal{S}_{K}(E, C)$ to $1_{\Theta\left(s, D_{s^{*}}\right)}$ for each $s \in S(E, C)$.

Proof. Since $S(E, C)$ is $E^{*}$-unitary (Proposition 2.19), it is a Hausdorff inverse semigroup. Hence the result follows from Theorem 4.2 and Theorems 4.12 and 4.13.

Corollary 4.15. Let $(E, C)$ be an adaptable separated graph, $S(E, C)$ be the inverse semigroup associated to $(E, C)$. Let $C^{*}(S(E, C))$ be the $C^{*}$-algebra universal for generators $E^{0} \cup E^{1} \cup\left\{\left(t_{i}^{v}\right)^{ \pm}: i \in \mathbb{N}, v \in E^{0}\right\}$ and the relations 2.2. Then there is an isomorphism

$$
C^{*}(S(E, C)) \cong C^{*}\left(\mathcal{G}_{\text {tight }}(S(E, C))\right)
$$

sending $\iota(s) \in C^{*}(S(E, C))$ to $1_{\Theta\left(s, D_{s^{*}}\right)}$ for each $s \in S(E, C)$.

Proof. The proofs of [32, Theorem 3.2.2 and Lemma 3.2.3] are easily modified to show that $C^{*}\left(\mathcal{G}_{\text {tight }}(S(E, C))\right.$ is universal for ${ }^{*}$-representations of $A_{\mathbb{C}}\left(\mathcal{G}_{\text {tight }}(S(E, C))\right)$. By definition, $C^{*}(S(E, C))$ is universal for ${ }^{*}$-representations of $\mathcal{S}_{\mathbb{C}}(E, C)$. So the result follows from Theorem 4.14.

\section{A NeW Description of the Groupoid $\mathcal{G}_{\text {tight }}(S(E, C))$}

In this section, we obtain a more concrete description of the tight groupoid $\mathcal{G}_{\text {tight }}(S(E, C))$ defined in the last section, paralleling the well-known description of graph groupoids (see, for example, [15]).

To this end, let $\hat{\mathcal{E}}_{\infty}$ be the space of infinite paths, described above (see Section 3), and consider the additive group $\Gamma:=\mathbb{Z}^{(\infty)} \times \mathbb{Z}^{(\infty)}$. Here, the first copy of $\mathbb{Z}^{(\infty)}$ will encode the 
exponents of the indeterminates $t_{i}^{v}$ and the second copy of $\mathbb{Z}^{(\infty)}$ will encode the difference of lengths between initial segments of infinite paths.

Our new approach to $\mathcal{G}_{\text {tight }}(S(E, C))$ is based on understanding the groupoid we subsequently define. First, if $\gamma$ is an initial segment ending in $E_{p}$, we define $|\gamma|_{\infty} \in \mathbb{Z}^{(\infty)}$ as follows: if $p$ is a regular, then $|\gamma|_{\infty}:=(|\gamma|, 0,0, \ldots,) \in \mathbb{Z}^{(\infty)}$; and, if $p$ is a free prime, then, expressing $\gamma=\gamma^{\prime} \prod_{j=1}^{k(p)} \alpha(p, j)^{l_{j}}$ where $\gamma^{\prime}$ is a finite path and $l_{j} \in \mathbb{Z}^{+}$, then $|\gamma|_{\infty}:=$ $\left(l_{1}+\left|\gamma^{\prime}\right|, \ldots, l_{k(p)}+\left|\gamma^{\prime}\right|, 0,0, \ldots\right)$.

Now consider the set $\mathcal{H}$ of triples $(x, n, y)$ such that

(i) $x$ and $y$ are infinite paths ending at the same prime $p$ of $I$; say $x=\gamma \lambda$, and $y=\nu \lambda$, where $\gamma$ and $\nu$ are initial segments ending in $E_{p}$, and $\lambda$ is an infinite path in $E_{p}$ (see Definitions 3.2 and 3.3); and

(ii) $n=\left(n_{1},|\gamma|_{\infty}-|\nu|_{\infty}\right) \in \Gamma$ for some $n_{1} \in \mathbb{Z}^{(\infty)}$.

We define $\mathcal{H}^{(2)}=\{((w, m, x),(y, n, z)) \in \mathcal{H} \times \mathcal{H}: x=y\}$, and we define multiplication from $\mathcal{H}^{(2)}$ to $\mathcal{H}$ by

$$
(x, n, y)(y, m, z)=(x, n+m, z) .
$$

Then $\mathcal{H}$ is a groupoid, with inverses given by $(x, n, y)^{-1}=(y,-n, x)$, and units $\mathcal{H}^{(0)}=$ $\left\{(x, 0, x): x \in \hat{\mathcal{E}}_{\infty}\right\}$ identified with $\hat{\mathcal{E}}_{\infty}$.

We now introduce a topology in $\mathcal{H}$. A basis of this topology is indexed by the elements in the inverse semigroup $S$.

Definition 5.1. Given $s \in S(E, C)$, written in the standard notation as $s=\gamma \mathbf{m}(p) \nu^{*}$, for finite paths $\gamma, \nu$ and monomial $\mathbf{m}(p)$ at $p \in I$, define $\mathcal{Z}(s)$ as the set of elements $(x, n, y) \in \mathcal{H}$ which satisfy the following conditions:

(1) If $p$ is a regular prime, then $\mathbf{m}(p)=\prod\left(t_{i}^{v}\right)^{d_{i}} \lambda \eta^{*}$, for paths of finite length $\lambda, \eta$ in $E_{p}$, with $s(\lambda)=v \in E_{p}^{0}$ and $r(\lambda)=r(\eta)$. In this case, $x=\gamma \lambda x_{0}$ and $y=\nu \eta x_{0}$, for an infinite path $x_{0}=\gamma^{\prime} \lambda^{\prime}$ starting at $r(\lambda)$, where $\gamma^{\prime}$ is a finite path and $\lambda^{\prime}$ is an infinite path in the component corresponding to $r\left(\gamma^{\prime}\right)$. Moreover, $n=\left(n_{1}, n_{2}\right)$, where $n_{1} \in$ $\mathbb{Z}^{(\infty)}$ is the sequence of exponents of $\phi_{\gamma^{\prime}}(\mathbf{m}(p))$ and $n_{2}=\left|\gamma \lambda \gamma^{\prime}\right|_{\infty}-\left|\nu \eta \gamma^{\prime}\right|_{\infty} \in \mathbb{Z}^{(\infty)}$. (Observe that $n_{2}$ is determined by the integer $|\gamma|+|\lambda|-|\nu|-|\eta|$ ).

(2) If $p$ is a free prime, then $\mathbf{m}(p)=\prod\left(t_{i}^{v^{p}}\right)^{d_{i}} \prod_{j=1}^{k(p)} \alpha(p, j)^{k_{j}}\left(\alpha(p, j)^{*}\right)^{l_{j}}$. In this case, either $x=\gamma \prod_{j=1}^{k(p)} \alpha(p, j)^{\infty}, y=\nu \prod_{j=1}^{\infty} \alpha(p, j)^{\infty}$, and $n=\left(n_{1}, n_{2}\right)$, with $n_{1}=\left(d_{i}\right)_{i \in \mathbb{N}}$, $n_{2}=\left(k_{1}+|\gamma|-l_{1}-|\nu|, \ldots, k_{k(p)}+|\gamma|-l_{k(p)}-|\nu|, 0, \ldots\right)$, or there exists an infinite path $\gamma^{\prime} \lambda^{\prime}$, where $\gamma^{\prime}=\alpha(p, i)^{m} \beta(p, i, s) \cdots, 1 \leq i \leq k(p)$ is a non-trivial finite path and $\lambda^{\prime}$ is an infinite path in the component corresponding to $r\left(\gamma^{\prime}\right)$, such that

$$
x=\gamma \alpha(p, i)^{k_{i}} \gamma^{\prime} \lambda^{\prime}, \quad y=\nu \alpha(p, i)^{l_{i}} \gamma^{\prime} \lambda^{\prime},
$$

and $n=\left(n_{1}, n_{2}\right)$, with $n_{1}$ equal to the sequence of exponents of $\phi_{\gamma^{\prime}}(\mathbf{m}(p))$ and $n_{2}=$ $\left|\gamma \alpha(p, i)^{k_{i}} \gamma^{\prime}\right|_{\infty}-\left|\nu \alpha(p, i)^{l_{i}} \gamma^{\prime}\right|_{\infty} \in \mathbb{Z}^{(\infty)}$. (Note that $n_{2}$ is determined by the integer $\left.|\gamma|-|\nu|+k_{i}-l_{i}\right)$.

Remark 5.2. Notice that when $e=\gamma \mathbf{m}(p) \gamma^{*}$ is an idempotent in $S(E, C)$, then

(a) if $p$ is a free prime, then $\mathbf{m}(p)=\prod_{j=1}^{k(p)} \alpha(p, j)^{k_{j}}\left(\alpha(p, j)^{*}\right)^{k_{j}}$, and $\mathcal{Z}(e)$ is the set of elements $(x,(0,0), x) \in \mathcal{H}$ such that $\lambda \prod_{j=1}^{k(p)} \alpha(p, j)^{k_{j}}$ is an initial segment of $x$; and 
(b) if $p$ is a regular prime, then $\mathbf{m}(p)=\lambda \lambda^{*}$ for a path of finite length $\lambda$ in the graph $E_{p}$, and $\mathcal{Z}(e)$ is the set of elements $(x,(0,0), x) \in \mathcal{H}$ such that $\gamma \lambda$ is an initial segment of $x$.

Therefore, using the identification of ultrafilters with infinite paths given in Corollary 3.5, we notice that $\mathcal{Z}(e)$ may be identified with the set $\mathcal{Z}(e)$ of Definition 3.7.

We now establish the isomorphism between the groupoid of germs of the action of $S(E, C)$ on $\hat{\mathcal{E}}_{\infty}$ and the groupoid $\mathcal{H}$.

Theorem 5.3. Let $(E, C)$ be an adaptable separated graph. Then there is a canonical isomorphism

$$
\phi: \mathcal{G}_{\text {tight }}(S(E, C)) \rightarrow \mathcal{H}
$$

between the topological groupoid $\mathcal{G}_{\text {tight }}(S(E, C))$ and the topological groupoid $\mathcal{H}$ defined above.

Proof. We start by observing that the elements of $\mathcal{G}_{\text {tight }}(S(E, C))$ can be represented by classes $[s, x]$, where $(s, x)$ is of a special form. Recall that $x$ is a point in the space $\hat{\mathcal{E}}_{\infty}$, and that $s$ is an element in $S(E, C)$ such that $s^{*} s \in x$ (interpreting $x$ as a subset of $\mathcal{E}$ ). Let us describe the structure of $x$ by cases:

(1) Assume first that $x=\nu \lambda$, where $\nu$ is a finite path and $\lambda$ is an infinite path in the component of a regular prime $p$. Then, we can assume that $s=\gamma\left(\prod\left(t_{i}^{v}\right)^{d_{i}}\right) \lambda^{\prime}\left(\lambda^{\prime \prime}\right)^{*} \nu^{*}$, where $\gamma$ is a finite path, $r(\gamma)=s\left(\lambda^{\prime}\right)=v \in E_{p}^{0}, r\left(\lambda^{\prime}\right)=r\left(\lambda^{\prime \prime}\right)$ and $\lambda^{\prime \prime}$ is an initial segment of $\lambda$, that is, $\lambda=\lambda^{\prime \prime} x_{0}$ for an infinite path $x_{0}$. In this case, we set

$$
\phi([s, x])=\left(\gamma \lambda^{\prime} x_{0},\left(\left(d_{i}\right)_{i \in \mathbb{N}},\left(|\gamma|+\left|\lambda^{\prime}\right|-\left|\lambda^{\prime \prime}\right|-|\nu|, 0, \ldots\right)\right), \nu \lambda^{\prime \prime} x_{0}\right) \in \mathcal{H} .
$$

(2) Assume now that $x=\nu \prod_{j=1}^{k(p)} \alpha(p, j)^{\infty}$ for a free prime $p$, where $\nu$ is a finite path ending at $v^{p}$. Then, $s$ can be chosen of the form

$$
s=\gamma\left(\prod\left(t_{i}^{v^{p}}\right)^{d_{i}}\right) \prod_{j=1}^{k(p)} \alpha(p, j)^{k_{j}}\left(\alpha(p, j)^{*}\right)^{l_{j}} \nu^{*}
$$

where $\gamma$ is a finite path ending at $v^{p}$. For an element $[s, x]$ of this form, we define

$$
\phi([s, x])=\left(\gamma \prod_{j=1}^{k(p)} \alpha(p, j)^{\infty},\left(\left(d_{i}\right)_{i \in \mathbb{N}},\left(|\gamma|+k_{j}-l_{j}-|\nu|\right)_{j}\right), \nu \prod_{j=1}^{k(p)} \alpha(p, j)^{\infty}\right) \in \mathcal{H} .
$$

In both cases (1) and (2), we can achieve the representative of the desired form by replacing the given element $[s, x]$ with a suitable element of the form $[s f, x]$, where $f \in \mathcal{E}$.

Notice that $\phi$ is a well-defined map. Indeed, if $[s, x]=[t, y]$, then $x=y$ and there exists $f \in x$ (thinking $x$ as a subset of $\hat{\mathcal{E}}_{\infty}$ ) such that $s f=t f$. In the free case, we can assume that $f=\nu \prod \alpha(p, j)^{t_{j}}\left(\alpha(p, j)^{*}\right)^{t_{j}} \nu^{*}$, where $t_{j}$ is bigger or equal than the corresponding powers of $\alpha(p, j)^{*}$ in $s$ and $t$. Hence, the elements $[s, x]$ and $[t, x]$ have the same image under $\phi$. The regular case is analogous.

It is straightforward to check that $\phi$ is an algebraic morphism of groupoids. Moreover, by the definition of $\mathcal{H}, \phi$ is surjective. Let us check that $\phi$ is injective. To this end, let us firstly work the free prime case out. Assume that $x=\nu \prod_{j=1}^{k(p)} \alpha(p, j)^{\infty}$ for a free prime $p$ and, fixing 
notation, that $s, t \in S(E, C)$, with $s$ as in (5.1) and

$$
t=\gamma^{\prime}\left(\prod\left(t_{i}^{v^{p}}\right)^{d_{i}^{\prime}}\right) \prod_{j=1}^{k(p)} \alpha(p, j)^{k_{j}^{\prime}}\left(\alpha(p, j)^{*}\right)^{l_{j}^{\prime}} \nu^{*} .
$$

If $\phi([s, x])=\phi([t, x])$, then $\gamma=\gamma^{\prime}, d_{i}=d_{i}^{\prime}$ for all $i$ and $k_{j}-l_{j}=k_{j}^{\prime}-l_{j}^{\prime}$ for all $1 \leq j \leq k(p)$. But then $k_{j}+l_{j}^{\prime}=k_{j}^{\prime}+l_{j}$ for all $j$, and so the element $f=\nu \prod_{j=1}^{k(p)} \alpha(p, j)^{k_{j}+l_{j}^{\prime}}\left(\alpha(p, j)^{*}\right)^{k_{j}^{\prime}+l_{j}} \nu^{*}$ is a idempotent in $S(E, C)$ such that $f \in x$ and $s f=t f$, which implies that $[s, x]=[t, x]$. The case where $x$ is an infinite path ending in a regular prime is left to the reader. Therefore, we conclude that $\phi$ is a bijective isomorphism of algebraic groupoids.

It remains only to show that the map $\phi$ is a homeomorphism. Recall that the topology of $\mathcal{G}:=\mathcal{G}_{\text {tight }}(S(E, C))$ is generated by the sets

$$
\Theta(s, U):=\{[s, x] \in \mathcal{G} \mid x \in U\},
$$

where $U$ is an open subset of $\hat{\mathcal{E}}_{\infty}$ such that $U \subseteq D_{s^{*} s}:=\left\{x \in \hat{\mathcal{E}}_{\infty} \mid s^{*} s \in x\right\}$. Given such an open set $U$, we know from Corollary 3.10 that $U=\bigcup_{f \in Z_{U}} \mathcal{Z}(f)$, where $Z_{U}$ is the set of idempotents $f \in \mathcal{E}$ such that $\mathcal{Z}(f) \subseteq U$, and thus

$$
\Theta(s, U)=\bigcup_{f \in Z_{U}} \Theta(s f, \mathcal{Z}(f))
$$

Therefore, the topology of $\mathcal{G}$ is generated by the open sets

$$
U_{s}:=\Theta\left(s, \mathcal{Z}\left(s^{*} s\right)\right)=\left\{[s, x] \mid s^{*} s \in x\right\},
$$

for $s \in S$. It is straightforward to check that $\phi\left(U_{s}\right)=\mathcal{Z}(s)$ for each $s \in S(E, C)$. This shows that $\phi$ is a homeomorphism, and the proof is complete.

Summing up, we obtain the following result.

Theorem 5.4. Let $(E, C)$ be an adaptable separated graph, and $S(E, C)$ be the inverse semigroup associated to $(E, C)$. Let $\mathcal{G}_{\text {tight }}(S(E, C))$ be the groupoid of germs associated to the canonical action of $S(E, C)$ on the space of ultrafilters $\hat{\mathcal{E}}_{\infty}$, and let $\mathcal{H}$ be the groupoid defined above. Then $\mathcal{G}_{\text {tight }}(S(E, C)) \cong \mathcal{H}$ is an ample Hausdorff étale topological groupoid.

Proof. By Theorem 5.3, there is an isomorphism of topological groupoids $\mathcal{G}_{\text {tight }}(S(E, C)) \cong \mathcal{H}$. By [17, Proposition 4.17], the groupoid $\mathcal{G}_{\text {tight }}(S(E, C))$ is an étale topological groupoid. Since $S(E, C)$ is $E^{*}$-unitary by Proposition 2.19, it follows from [17, Propositions 6.4 and 6.2] that $\mathcal{G}_{\text {tight }}(S(E, C))$ is Hausdorff. Finally, observe that $U_{s}=\Theta\left(s, \mathcal{Z}\left(s^{*} s\right)\right), s \in S(E, C)$, is a basis of compact open bisections for the topology of $\mathcal{G}_{\text {tight }}(S(E, C))$.

\section{Amenability of $\mathcal{G}_{\text {tight }}(S(E, C))$}

In this section we show that the groupoid $\mathcal{G}_{\text {tight }}(S(E, C))$ associated to any adaptable separated graph is amenable (Proposition 6.2). Thus the full and reduced $C^{*}$-algebras of this groupoid coincide, are nuclear, and belong to the UCT class. In particular, Corollary 4.15 shows that $C_{r}^{*}\left(\mathcal{G}_{\text {tight }}(S(E, C))\right.$ is universal for tight representations of $S(E, C)$, so $C^{*}(S(E, C))$ has a faithful representation on $\bigoplus_{x \in \widehat{\mathcal{E}}^{\infty}} \ell^{2}\left(G_{\text {tight }}(S(E, C))_{x}\right)$. These results open the possibility of addressing the realisation problem via nuclear $C^{*}$-algebras in the UCT class. 
The first step is to reduce the question to dealing with the groupoid sitting over any prime $p \in I$, because this significantly simplifies the question. To do this, we will use the following general lemma, together with the fact that, since $I$ is finite, the groupoid admits a finite "composition series." In the following result, given a poset $\mathcal{O}$ and an element $V \in \mathcal{O}$ we will say that $U \in \mathcal{O}$ is subjacent to $V$ if $U$ is a maximal element of the set $\{W \in \mathcal{O} \backslash\{V\}: W \preceq V\}$.

Lemma 6.1. Let $\mathcal{G}$ be a second-countable Hausdorff étale groupoid. Suppose that $\mathcal{O}$ is a finite collection of open invariant subsets of $\mathcal{G}^{(0)}$ that is closed under intersections. Suppose that $\mathcal{G}^{(0)}$ and $\emptyset$ both belong to $\mathcal{O}$, and regard $\mathcal{O}$ as a poset under $\subseteq$. Suppose that, whenever $U$ is subjacent to $V$ in $\mathcal{O}$, the groupoid $\left.\mathcal{G}\right|_{V \backslash U}$ is amenable. Then, $\mathcal{G}$ is amenable.

Proof. We proceed by induction on $|\mathcal{O}|$. First, suppose that $|\mathcal{O}|=1$. Since $\mathcal{G}^{(0)}$ and $\emptyset$ both belong to $\mathcal{O}$, we deduce that $\mathcal{G}^{(0)}=\emptyset$, and so $\mathcal{G}=\emptyset$ is trivially amenable.

Now fix $n \geq 1$. Suppose as an inductive hypothesis that the result holds for groupoids in which $|\mathcal{O}| \leq n$, and suppose that $|\mathcal{O}|=n+1$. Let $V$ be a maximal element of $\mathcal{O} \backslash\left\{\mathcal{G}^{(0)}\right\}$. By hypothesis, $\left.\mathcal{G}\right|_{\mathcal{G}^{(0)} \backslash V}$ is amenable. Let $\mathcal{O}^{\prime}:=\{U \cap V: U \in \mathcal{O}\}$. Since $\mathcal{O}$ is closed under intersections, we have $\mathcal{O}^{\prime}=\{W \in \mathcal{O}: W \subseteq V\} \subseteq \mathcal{O}$, and in particular if $U$ is subjacent to $U^{\prime}$ in $\mathcal{O}^{\prime}$ then $U$ is subjacent to $U^{\prime}$ in $\mathcal{O}$. So $\mathcal{O}^{\prime}$ is a finite collection of open invariant subsets of $\left.\mathcal{G}\right|_{V} ^{(0)}$ that is closed under intersections and contains $\left.\mathcal{G}\right|_{V} ^{(0)}$ and $\emptyset$, and whenever $U$ is subjacent to $U^{\prime}$ in $\mathcal{O}^{\prime}$ the groupoid $\left(\left.\mathcal{G}\right|_{V}\right)_{U^{\prime} \backslash U}=\left.\mathcal{G}\right|_{U^{\prime} \backslash U}$ is amenable. Since $V \cap V=\mathcal{G}^{(0)} \cap V$, we see that $\left|\mathcal{O}^{\prime}\right| \leq|\mathcal{O}|-1=n$. So the inductive hypothesis implies that $\left.\mathcal{G}\right|_{V}$ is amenable. Thus [37, Proposition 9.82] shows that $\mathcal{G}$ is amenable.

Proposition 6.2. Let $(E, C)$ be an adaptable separated graph. The groupoid $\mathcal{G}_{\text {tight }}(S(E, C))$ is amenable.

Proof. Let $\mathcal{H}$ be the groupoid, isomorphic to $\mathcal{G}_{\text {tight }}(S(E, C))$, described in Section 5. We say that a subset $P \subseteq I$ is hereditary if $p \in P$ and $p \geq q$ implies $q \in P$. For each hereditary $P \subseteq I$, the set

$$
W_{P}:=\bigcup_{p \in P}\left\{\gamma \lambda \in \mathcal{H}^{(0)}: \gamma \text { is an initial segment, } r(\gamma) \in E_{p}^{0} \text { and } \lambda \in E_{p}^{\infty}\right\}
$$

is an open invariant subset of $\mathcal{H}^{(0)}$. Clearly, the collection $\mathcal{O}=\left\{W_{P}: P \subseteq I\right.$ is hereditary $\}$ is a finite collection of open invariant sets that contains $\emptyset=W_{\emptyset}$ and $\mathcal{H}^{(0)}=W_{I}$, and is closed under intersections since $W_{P} \cap W_{Q}=W_{P \cap Q}$.

If $P, Q$ are hereditary subsets of $I$, then $W_{P} \subseteq W_{Q}$ if and only if $P \subseteq Q$. Suppose that $U, V \in \mathcal{O}$, and that $U$ is subjacent to $V$ in $\mathcal{O}$. Then $V=W_{Q}$ and $U=W_{P}$ for some nested pair $P \subsetneq Q$ of hereditary subsets of $I$. Since $Q \backslash P$ is nonempty, we deduce that it contains a maximal element $q \in Q$. Hence $Q^{\prime}=Q \backslash\{q\}$ is a proper hereditary subset of $Q$ containing $P$. Thus $Q^{\prime}=P$, forcing $Q \backslash P=\{q\}$. So by Lemma 6.1 it suffices to show that if $P, Q$ are hereditary subsets of $I$, with $P \subset Q$, and $Q \backslash P=\{q\}$ is a singleton, then $\left.\mathcal{H}\right|_{W_{Q} \backslash W_{P}}$ is amenable.

Fix such a $P, Q$ and $q$. Then,

$$
W_{Q} \backslash W_{P}=\left\{\gamma \lambda \in \mathcal{H}^{(0)}: \gamma \text { is an initial segment, } r(\gamma) \in E_{q}^{0} \text { and } \lambda \in E_{q}^{\infty}\right\}
$$

The subset $X_{q}:=\left\{\lambda \in \mathcal{H}^{(0)}: \lambda\right.$ is an infinite path in $\left.E_{q}\right\}$ is a full open subset of the unit space of $\left.\mathcal{H}\right|_{W_{Q} \backslash W_{P}}$. Hence, the final statement of [19, Theorem 3.10] shows that $\left.\mathcal{H}\right|_{W_{Q} \backslash W_{P}}$ is equivalent in the sense of [25, Definition 2.1] (see also [30, Section 3]) to the groupoid 
$\left.\mathcal{H}\right|_{X_{q}}$. Since amenability is preserved by groupoid equivalence [2, Theorem 2.2.17], it therefore suffices to show that each $\left.\mathcal{H}\right|_{X_{q}}$ is amenable.

By definition, we have $\left.\mathcal{H}\right|_{X_{q}}=\left\{\left(\gamma \lambda,\left(n_{1},|\gamma|_{\infty}-|\nu|_{\infty}\right), \nu \lambda\right): \gamma, \nu \in E_{q}^{*}, \lambda \in E_{q}^{\infty}, n_{1} \in \mathbb{Z}^{(\infty)}\right\}$, and the map $c:\left(\gamma \lambda,\left(n_{1},|\gamma|_{\infty}-|\nu|_{\infty}\right), \nu \lambda\right) \mapsto n_{1}$ is a continuous cocycle from $\left.\mathcal{H}\right|_{X_{q}}$ to the countable abelian group $\mathbb{Z}^{(\infty)}$. Thus [33, Proposition 9.3] implies that $\left.\mathcal{H}\right|_{X_{q}}$ is amenable provided that $c^{-1}(0)$ is amenable. We have $c^{-1}(0) \cong\left\{\left(\gamma \lambda,|\gamma|_{\infty}-|\nu|_{\infty}, \nu \lambda\right): \gamma, \nu \in E_{q}^{*}, \lambda \in\right.$ $\left.E_{q}^{\infty}\right\}$. If $q$ is a regular prime, this is precisely the graph groupoid $\mathcal{G}_{E_{q}}$, which is amenable by [22, Corollary 5.5]; and if $q$ is a free prime, then $\gamma$ and $\nu$ are finite products of the $\alpha(q, j)$, and the map $\left(\gamma \lambda,|\gamma|_{\infty}-|\nu|_{\infty}, \nu \lambda\right) \mapsto|\gamma|_{\infty}-|\nu|_{\infty}$ is an isomorphism of $\left.\mathcal{H}\right|_{X_{q}}$ onto $\mathbb{Z}^{k(q)}$, which again is amenable. So in either case $\left.\mathcal{H}\right|_{X_{q}}$ is amenable, completing the proof.

\section{The TYPe SEMigroup}

7.1. The type semigroup of a Boolean inverse semigroup. Let $S$ be an inverse semigroup (always with 0 ). We denote by $\mathcal{E}(S)$ the semilattice of idempotents of $S$. We say that $x, y \in S$ are orthogonal, written $x \perp y$ if $x^{*} y=y x^{*}=0$

Recall that a Boolean inverse semigroup is an inverse semigroup $S$ such that $\mathcal{E}(S)$ is a Boolean ring (a ring with $x=x^{2}$ for all $x$ ), and such that every pair $x, y \in S$ satisfying $x \perp y$ has a supremum, denoted $x \oplus y \in S$ (see [36, Definition 3-1.6] for further details).

Definition 7.1. [36, page 98] Let $S$ be a Boolean inverse semigroup. The type semigroup (or type monoid) of $S$ is the commutative monoid $\operatorname{Typ}(S)$ freely generated by elements typ $(x)$, where $x \in \mathcal{E}(S)$, subject to the relations

(1) $\operatorname{typ}(0)=0$

(2) $\operatorname{typ}(x)=\operatorname{typ}(y)$ whenever $x, y \in \mathcal{E}(S)$ and there is $s \in S$ such that $s^{*}=x$ and $s^{*} s=y$.

(3) $\operatorname{typ}(x \oplus y)=\operatorname{typ}(x)+\operatorname{typ}(y)$ whenever $x, y$ are orthogonal elements in $\mathcal{E}(S)$.

By [36, Corollary 4-1.4], Typ $(S)$ is a conical refinement monoid.

7.2. The type semigroup of an ample groupoid. Let $\mathcal{G}$ be a (not-necessarily-Hausdorff) étale groupoid, with a Hausdorff locally compact unit space $X:=\mathcal{G}^{(0)}$. Then the collection $S(\mathcal{G})$ of all compact open bisections of $\mathcal{G}$ forms a Boolean inverse semigroup. Indeed, it is well-known that it is an inverse semigroup, and since the topology on $X$ is Hausdorff, one obtains that the semilattice of idempotents of $S(\mathcal{G})$, which coincides with the set of all the compact open subsets of $X$, is a Boolean ring. Moreover, if we have two orthogonal compact open bisections, it is clear that their disjoint union is again a compact open bisection.

Definition 7.2. Let $\mathcal{G}$ be a (non-necessarily Hausdorff) étale groupoid, with a Hausdorff locally compact base space $X:=\mathcal{G}^{(0)}$. We define the type semigroup of $\mathcal{G}$ as

$$
\operatorname{Typ}(\mathcal{G})=\operatorname{Typ}(S(\mathcal{G}))
$$

If $\mathcal{G}$ is second countable, then the type semigroup $\operatorname{Typ}(\mathcal{G})$ is a countable conical refinement monoid.

Now, we will show that $\operatorname{Typ}(\mathcal{G})$ coincides with some recently defined type semigroups $[12,28,29]$. To this end, we recall the definition in [12] (which is equivalent to the ones given in [28] and [29], as shown in [29]). 
Let $\mathcal{G}$ be an ample Hausdorff groupoid. Define an equivalence relation $\sim$ on the set

$$
\left\{\bigcup_{i=1}^{n} A_{i} \times\{i\} \mid n \in \mathbb{N}, A_{i} \in \mathcal{E}(S(\mathcal{G}))\right\}
$$

as follows: for $A=\bigcup_{i=1}^{n} A_{i} \times\{i\}$ and $B=\bigcup_{j=1}^{m} B_{j} \times\{j\}$, write $A \sim B$ if there exist $l \in \mathbb{N}$, open compact bisections $W_{1}, \ldots, W_{l}$, and $n_{1}, \ldots, n_{l}, m_{1}, \ldots, m_{l} \in \mathbb{N}$ such that

$$
A=\bigsqcup_{k=1}^{l} d\left(W_{k}\right) \times\left\{n_{k}\right\}, \quad B=\bigsqcup_{k=1}^{l} r\left(W_{k}\right) \times\left\{m_{k}\right\} .
$$

The type semigroup of $\mathcal{G}$ is then defined in [12] to be

$$
S\left(\mathcal{G}, \mathcal{G}^{a}\right)=\left\{\bigcup_{i=1}^{n} A_{i} \times\{i\} \mid n \in \mathbb{N}, A_{i} \in \mathcal{E}(S(\mathcal{G}))\right\} / \sim .
$$

Proposition 7.3. Let $\mathcal{G}$ be an ample Hausdorff groupoid. Then $\operatorname{Typ}(\mathcal{G}) \cong S\left(\mathcal{G}, \mathcal{G}^{a}\right)$.

Proof. There is a well-defined monoid homomorphism

$$
\Phi: \operatorname{Typ}(\mathcal{G}) \rightarrow S\left(\mathcal{G}, \mathcal{G}^{a}\right)
$$

defined by $\Phi([A])=[A \times\{1\}]$, for every compact open subset $A$ of $\mathcal{G}^{0}$. Since $[A \times\{1\}]=$ $[A \times\{i\}]$ in $S\left(\mathcal{G}, \mathcal{G}^{a}\right)$, we easily see that this map is surjective. To show it is injective, assume that $\Phi\left(\sum_{i=1}^{n}\left[A_{i}\right]\right)=\Phi\left(\sum_{j=1}^{m}\left[B_{j}\right]\right)$ for compact open subsets $A_{i}, B_{j}$ of $\mathcal{G}^{0}$. Then

$$
A:=\bigcup_{i=1}^{n} A_{i} \times\{i\} \sim B:=\bigcup_{j=1}^{m} B_{j} \times\{j\}
$$

so there are open compact bisections $W_{1}, \ldots, W_{l}$, and elements $n_{1}, \ldots, n_{l}, m_{1}, \ldots, m_{l} \in \mathbb{N}$ such that

$$
A=\bigsqcup_{k=1}^{l} d\left(W_{k}\right) \times\left\{n_{k}\right\}, \quad B=\bigsqcup_{k=1}^{l} r\left(W_{k}\right) \times\left\{m_{k}\right\} .
$$

It follows that there is a partition $\{1, \ldots, l\}=\bigsqcup_{i=1}^{n} I_{i}$ such that for each $i \in\{1 \ldots, n\}$ we have $A_{i}=\bigsqcup_{j \in I_{i}} d\left(W_{j}\right)$. We thus get in $\operatorname{Typ}(\mathcal{G})$

$$
\sum_{i=1}^{n}\left[A_{i}\right]=\sum_{i=1}^{n} \sum_{j \in I_{i}}\left[d\left(W_{j}\right)\right]=\sum_{i=1}^{n} \sum_{j \in I_{i}}\left[r\left(W_{j}\right)\right]=\sum_{j=1}^{l}\left[r\left(W_{j}\right)\right]=\sum_{j=1}^{m}\left[B_{j}\right],
$$

showing injectivity.

Let us now move to the framework of these notes. Namely, let $(E, C)$ be an adaptable separated graph, and consider the ample Hausdorff groupoid $\mathcal{G}_{\text {tight }}(S(E, C))$.

We want to relate the type semigroup of $\mathcal{G}_{\text {tight }}(S(E, C))$ with our original primely generated refinement monoid $M(E, C)$ (see Section 1.2). To do this, we need the next lemma. Recall that the notation $\mathcal{Z}(s)$, for $s \in S(E, C)$, has been introduced in Definition 5.1, and that the family $\{\mathcal{Z}(s)\}_{s \in S(E, C)}$ is a basis of open compact bisections of $\mathcal{H}$. Since $\mathcal{G}_{\text {tight }}(S(E, C)) \cong$ $\mathcal{H}$ with an isomorphism sending $U_{s}$ onto $\mathcal{Z}(s)$, we will identify these two groupoids, and thus consider $\mathcal{Z}(s)$ as open compact subsets of $\mathcal{G}_{\text {tight }}(S(E, C))$. Under this identification, Definition 3.7 and Definition 5.1 agree on $\mathcal{Z}(e)$ for any idempotent $e \in \mathcal{E}$. 
Lemma 7.4. The following properties hold for the groupoid $\mathcal{G}_{\text {tight }}(S(E, C))$.

(1) Suppose that $e, e_{1}, \ldots, e_{N} \in \mathcal{E}$. Then

$$
\mathcal{Z}(e)=\bigsqcup_{i=1}^{N} \mathcal{Z}\left(e_{i}\right)
$$

if and only if $\left\{e_{1}, \ldots e_{N}\right\}$ is an irredundant finite cover of $e$.

(2) Given any compact open bisection $U$, we have

$$
U=\bigsqcup_{i=1}^{N} \mathcal{Z}\left(s_{i}\right),
$$

for suitable $s_{i} \in S, i=1, \ldots, N$.

Proof. (1) If $\left\{e_{1}, \ldots, e_{N}\right\}$ is an irredundant finite cover of $e$, then $\mathcal{Z}(e)=\bigsqcup_{i=1}^{N} \mathcal{Z}\left(e_{i}\right)$ because the representation $\iota: S(E, C) \rightarrow \mathcal{S}_{K}(E, C)$ is tight. Conversely, assume that $\mathcal{Z}(e)=$ $\bigsqcup_{i=1}^{N} \mathcal{Z}\left(e_{i}\right)$. Then the idempotents $e_{i}$ are mutually orthogonal. Now let $f$ be a non-zero idempotent such that $f \leq e$. Then $\mathcal{Z}(f)$ is a non-empty subset of $\mathcal{Z}(e)$, and thus $\mathcal{Z}\left(f e_{i}\right)=$ $\mathcal{Z}(f) \cap \mathcal{Z}\left(e_{i}\right)$ is non-empty for some $i=1, \ldots, n$. This implies that $f e_{i} \neq 0$. Hence $\left\{e_{1}, \ldots e_{N}\right\}$ is an irredundant finite cover of $e$.

(2) Let $U$ be a compact open bisection. We have $U=\bigcup_{i=1}^{n} \mathcal{Z}\left(s_{i}\right)$ for elements $s_{i} \in$ $S(E, C)$. Now, observe that $s(U)=\bigcup_{i=1}^{n} s\left(\mathcal{Z}\left(s_{i}\right)\right)=\bigcup_{i=1}^{n} \mathcal{Z}\left(s_{i}^{*} s_{i}\right)$. By using the standard decomposition sketched in the proof of Lemma 3.9, we can write

$$
s(U)=\bigsqcup_{k=1}^{N} U_{k},
$$

a disjoint union of open compact subsets $U_{k}$ of $\hat{\mathcal{E}}_{\infty}$ such that each $U_{k}$ is contained in a (not necessarily unique) set $\mathcal{Z}\left(s_{i}^{*} s_{i}\right)$. Now, by using Corollary 3.10, we can write each $U_{k}$ as a disjoint union of sets of the form $\mathcal{Z}\left(e_{k, l}\right)$, for idempotents $e_{k, l} \in \mathcal{E}$. For each $k \in\{1, \ldots, N\}$, let $\tau(k) \in\{1, \ldots, n\}$ be such that $U_{k} \subseteq s\left(\mathcal{Z}\left(s_{\tau(k)}\right)\right)$. Then

$$
U=\bigsqcup_{k, l} \mathcal{Z}\left(s_{\tau(k)} e_{k, l}\right)
$$

because, since $U$ is a bisection, $s$ defines a homeomorphism $U \rightarrow s(U)$, sending $\mathcal{Z}\left(s_{\tau(k)} e_{k, l}\right)$ onto $\mathcal{Z}\left(e_{k, l}\right)$.

With this lemma, we are ready to show the main result of this paper.

Theorem 7.5. Let $(E, C)$ be an adaptable separated graph, $S(E, C)$ be the inverse semigroup associated to $(E, C)$, and let $\mathcal{G}_{\text {tight }}(S(E, C))$ be the groupoid of germs associated to the canonical action of $S(E, C)$ on the space of ultrafilters $\hat{\mathcal{E}}_{\infty}$. Then, there is a monoid isomorphism

$$
\psi: M=M(E, C) \rightarrow \operatorname{Typ}\left(\mathcal{G}_{\text {tight }}(S(E, C))\right)
$$

such that $\psi\left(a_{v}\right)=[\mathcal{Z}(v)]$ for every $v \in E^{0}$.

Proof. Since the defining relations in the semigroup $M(E, C)$ are generated by the expansions in Remark 4.3, Lemmas 4.10 and 7.4 show that these relations are preserved under $\psi$. Hence, we obtain a monoid homomorphism $\psi$ satisfying $\psi\left(a_{v}\right)=[\mathcal{Z}(v)]$ for every $v \in E^{0}$. 
Given an idempotent $e=\gamma \mathbf{m}(p) \gamma^{*}$ in $S(E, C)$, we obviously have $[\mathcal{Z}(e)]=[\mathcal{Z}(v)]$, where $v=v^{p}$ if $p$ is a free prime and $v=r(\lambda) \in E_{p}^{0}$ if $p$ is a regular prime and $\mathbf{m}(p)=\lambda \lambda^{*}$. It follows that the map $\psi$ is surjective.

We now show that $\psi$ is injective. Assume that $\psi\left(\sum_{i=1}^{n} a_{v_{i}}\right)=\psi\left(\sum_{j=1}^{m} a_{w_{j}}\right)$ in $\operatorname{Typ}(\mathcal{G})$. Using Proposition 7.3, we see that this implies that

$$
A:=\bigsqcup_{i=1}^{n} \mathcal{Z}\left(v_{i}\right) \times\{i\} \sim B:=\bigsqcup_{j=1}^{m} \mathcal{Z}\left(w_{j}\right) \times\{j\}
$$

in $S\left(\mathcal{G}, \mathcal{G}^{a}\right)$. Now, by the definition of $\sim$ and Lemma 7.4 , there exist $l \in \mathbb{N}, s_{1}, \ldots, s_{l} \in$ $S(E, C)$, and $n_{1}, \ldots n_{l}, m_{1}, \ldots, m_{l}$ such that

$$
A=\bigsqcup_{k=1}^{l} s\left(\mathcal{Z}\left(s_{k}\right)\right) \times\left\{n_{k}\right\}, \quad B=\bigsqcup_{k=1}^{l} r\left(\mathcal{Z}\left(s_{k}\right)\right) \times\left\{m_{k}\right\} .
$$

It follows that there are partitions $\{1, \ldots, l\}=\bigsqcup_{i=1}^{n} I_{i}$ and $\{1, \ldots, l\}=\bigsqcup_{j=1}^{m} J_{j}$ such that

$$
\mathcal{Z}\left(v_{i}\right)=\bigsqcup_{k \in I_{i}} s\left(\mathcal{Z}\left(s_{k}\right)\right) \quad \text { and } \quad \mathcal{Z}\left(w_{j}\right)=\bigsqcup_{k \in J_{j}} r\left(\mathcal{Z}\left(s_{k}\right)\right)
$$

for $i=1, \ldots, n$ and $j=1, \ldots, m$. But now, $s\left(\mathcal{Z}\left(s_{k}\right)\right)=\mathcal{Z}\left(s_{k}^{*} s_{k}\right)$, and $r\left(\mathcal{Z}\left(s_{k}\right)\right)=\mathcal{Z}\left(s_{k} s_{k}^{*}\right)$. Thus, $\mathcal{Z}\left(v_{i}\right)=\bigsqcup_{k \in I_{i}} \mathcal{Z}\left(s_{k}^{*} s_{k}\right)$, which by Lemma $7.4(1)$ implies that $\left\{s_{k}^{*} s_{k}\right\}_{k \in I_{i}}$ is an irredundant finite cover of $v_{i}$, for $i=1, \ldots, n$. It follows from Proposition 4.11 that $\left\{s_{k}^{*} s_{k}\right\}_{k \in I_{i}}$ is an expanded set of $v_{i}$, and thus that $a_{v_{i}}=\sum_{k \in I_{i}}\left[s_{k}^{*} s_{k}\right]$ for $i=1, \ldots, n$, where we denote by $\left[s_{k}^{*} s_{k}\right]$ the class in $M(E, C)$ of the vertex in $E$ representing the idempotent $s_{k}^{*} s_{k}$. Analogously, $a_{w_{j}}=\sum_{k \in J_{j}}\left[s_{k} s_{k}^{*}\right]$ for $j=1, \ldots, m$. Since $\left[s_{k}^{*} s_{k}\right]=\left[s_{k} s_{k}^{*}\right]$ in $M(E, C)$, we obtain

$$
\sum_{i=1}^{n} a_{v_{i}}=\sum_{i=1}^{n} \sum_{k \in I_{i}}\left[s_{k}^{*} s_{k}\right]=\sum_{k=1}^{l}\left[s_{k}^{*} s_{k}\right]=\sum_{k=1}^{l}\left[s_{k} s_{k}^{*}\right]=\sum_{j=1}^{m} \sum_{k \in J_{j}}\left[s_{k} s_{k}^{*}\right]=\sum_{j=1}^{m} a_{w_{j}},
$$

and so $\psi$ is injective.

The following corollary follows immediately from Theorems 1.4 and 7.5.

Corollary 7.6. Let $M$ be a finitely generated conical refinement monoid. Then there is an adaptable separated graph $(E, C)$ such that

$$
M \cong \operatorname{Typ}\left(\mathcal{G}_{\text {tight }}(S(E, C))\right) .
$$

In particular, all finitely generated conical refinement monoids arise as type semigroups of ample Hausdorff groupoids.

\section{ACKNOWLEDGMENTS}

This research project was initiated when the authors were at the Centre de Recerca Matemàtica as part of the Intensive Research Program Operator algebras: dynamics and interactions in 2017, and the work was significantly supported by the research environment and facilities provided there. We thank the Centre de Recerca Matemàtica for its support. 


\section{REFERENCES}

[1] G. Abrams, P. Ara, M. Siles Molina, Leavitt Path Algebras, Springer Lecture Notes in Mathematics, vol. 2191, 2017.

[2] C. Anantharaman-Delaroche, J. Renault, Amenable groupoids, Monographies de L'Enseignement Mathématique 36, L'Enseignement Mathématique, Geneva, (2000).

[3] P. ARA, The realization problem for von Neumann regular rings, in Ring Theory 200\%. Proceedings of the Fifth China-Japan-Korea Conference, (H. Marubayashi, K. Masaike, K. Oshiro, M. Sato, Eds.), Hackensack, NJ (2009) World Scientific, pp. 21-37.

[4] P. Ara, J. Bosa, E. PARdo, Refinement monoids and adaptable separated graphs, arXiv:1904.04255 [math.RA].

[5] P. Ara, J. Bosa, E. Pardo, The realization problem for finitely generated refinement monoids, in preparation.

[6] P. ArA, R. Exel, Dynamical systems associated to separated graphs, graph algebras, and paradoxical decompositions, Adv. Math. 252 (2014), 748-804.

[7] P. Ara, K.R. Goodearl, Leavitt path algebras of separated graphs, J. Reine Angew. Math. 669 (2012), 165-224.

[8] P. Ara, M.A. Moreno, E. Pardo, Nonstable K-Theory for graph algebras, Algebra Rep. Th. 10 (2007), 157-178.

[9] P. Ara, E. PArdo, Primely generated refinement monoids, Israel J. Math. 214 (2016), 379-419.

[10] P. Ara, E. Pardo, Representing finitely generated refinement monoids as graph monoids J. Algebra 480 (2017), 79-123.

[11] P Ara, F. Perera, F. Wehrung, Finitely generated antisymmetric graph monoids, J. Algebra 320 (2008), 1963-1982.

[12] C. Bönicke, K. LI, Ideal structure and pure infiniteness of ample groupoid $C^{*}$-algebras, Ergodic Theory and Dynamical Systems, 1-30. doi:10.1017/etds.2018.39.

[13] G. Brookfield, Cancellation in primely generated refinement monoids, Algebra Universalis 46 (2001), $343-371$.

[14] L.O. Clark, R. Exel, E. Pardo A generalized uniqueness theorem and the graded ideal structure of Steinberg algebras, Forum Math 30 (2017), 533-552.

[15] L.O. Clark, C. Farthing, A. Sims, M. Tomforde, A groupoid generalization of Leavitt path algebras, Semigroup Forum 89 (2014), 501-517.

[16] L.O. Clark, R. Hazrat, Étale groupoids and Steinberg algebras, a concise introduction, arXiv:1901.01612v1 [math.RA].

[17] R. Exel, Inverse semigroups and combinatorial $C^{*}$-algebras, Bull. Braz. Math. Soc. (N.S.) 39 (2008), 191-313.

[18] R. Exel, E. PARdo, The tight groupoid of an inverse semigroup, Semigroup Forum 92 (2016), 274-303.

[19] C. Farsi, A. Kumjian, D. Pask, A. Sims, Ample groupoids: equivalence, homology, and Matui's HK-conjecture, arXix:1808.07807 [math.OA].

[20] K.R. Goodearl, Von Neumann regular rings and direct sum decomposition problems, in "Abelian groups and modules" (Padova,1994), Math. and its Applics. 343, pp. 249-255, Kluwer Acad. Publ., Dordrecht (1995).

[21] D.G. Jones, M.V. Lawson, Graph inverse semigroups: their characterization and completion, $J$. Algebra 409 (2014), 444-473.

[22] A. Kumjian, D. Pask, I. Raeburn, J. Renault, Graphs, groupoids, and Cuntz-Krieger algebras, J. Funct. Anal. 144 (1997), 505-541.

[23] M.V. Lawson, "Inverse Semigroups, the Theory of Partial Symmetries", World Scientific, Singapore (1998).

[24] H. Matui, Étale groupoids arising from products of shifts of finite type, Adv. Math. 303 (2016), $502-548$.

[25] P.S. Muhly, J.N. Renault, D.P. Williams, Equivalence and isomorphism for groupoid $C^{*}$-algebras, J. Operator Theory 17 (1987), 3-22. 
[26] P. Nyland, E. Ortega, Topological full groups of ample groupoids with applications to graph algebras, arXiv:1806.11087v3 [math.OA].

[27] E. Ortega, Homology of the Exel-Katsura-Pardo groupoid, arXiv:1806.09297v2 [math.OA].

[28] D. Pask, A. Sierakowski, A. Sims, Unbounded quasitraces, stably finiteness and pure infiniteness, arXiv:1705.01268 [math.OA].

[29] T. Rainone, A. Sims, A dichotomy for groupoid $C^{*}$-algebras, arXiv:1707.04516 [math.OA].

[30] J. Renault, A groupoid approach to $C^{*}$-algebras, Lecture Notes in Mathematics 793, Springer, Berlin, 1980.

[31] S.W. RigBy, The groupoid approach to Leavitt path algebras. Preprint, 2018.

[32] A. Sims, Hausdorff étale groupoids and their $C^{*}$-algebras, to appear in Advanced Courses in Mathematics, Operator algebras and dynamics: groupoids, crossed products and Rokhlin dimension, Birkhäuser, CRM Barcelona, 2018.

[33] J. SpielberG, Groupoids and $C^{*}$-algebras for categories of paths, Trans. Amer. Math. Soc. 366 (2014), $5771-5819$.

[34] B. Steinberg, A groupoid approach to discrete inverse semigroup algebras, Adv. Math. 223 (2010), 689-727.

[35] B. Steinberg, Simplicity, primitivity and semiprimitivity of étale groupoid algebras with applications to inverse semigroup algebras, J. Pure Appl. Algebra 220 (2016), 1035-1054.

[36] F. Wehrung, Refinement monoids, equidecomposable types, and Boolean inverse semigroups, Springer Lecture Notes in Math. 2188 (2017).

[37] D.P. Williams, A Tool Kit for Groupoid $C^{*}$-algebras (in preparation).

Departament de Matemàtiques, Universitat Autònoma de Barcelona, 08193 Bellaterra (Barcelona), Spain, and Barcelona Graduate School of Mathematics (BGSMath).

E-mail address: para@mat.uab.cat

E-mail address: jbosa@mat.uab.cat

Departamento de Matemáticas, Facultad de Ciencias, Universidad de Cádiz, Campus de Puerto Real, 11510 Puerto Real (Cádiz), Spain.

E-mail address: enrique.pardo@uca.es

$U R L:$ https://sites.google.com/a/gm.uca.es/enrique-pardo-s-home-page/

School of Mathematics and Applied Statistics, University of Wollongong, Wollongong NSW 2522, Australia.

E-mail address: asims@uow.edu.au 